Supporting Information for

\title{
Simple, General and Efficient. Synthesis of Meso-substituted Borondipyrromethenes from a Single Platform
}

Eduardo Peña-Cabrera, * Angélica Aguilar-Aguilar, Martha González-Domínguez, Erik Lager, Rubí Zamudio-Vázquez, Jazmín Godoy-Vargas and Fabián Villanueva-García.

Facultad de Química, Universidad de Guanajuato, Col. Noria Alta S/N, Guanajuato, Gto. 36050 Mexico.

Fax: (+ 52) 473-732 0006 ext. 8111

eduardop@quijote.ugto.mx

\section{Table of Contents}

S2 ...............................General Section

S3-S6.............................. Procedures

S7 ................................References

S8-S55.............................. SMR Spectra 


\section{General Section}

${ }^{1} \mathrm{H}$ and ${ }^{13} \mathrm{C}$ NMR spectra were recorded on a Varian Gemini $200(200 \mathrm{MHz})$ spectrometer in deuteriochloroform $\left(\mathrm{CDCl}_{3}\right)$ with either tetramethylsilane (TMS) $(0.00$ ppm ${ }^{1} \mathrm{H}, 0.00 \mathrm{ppm}{ }^{13} \mathrm{C}$ ) or chloroform $\left(7.26 \mathrm{ppm}{ }^{1} \mathrm{H}, 77.00 \mathrm{ppm}{ }^{13} \mathrm{C}\right)$ or as internal reference unless otherwise stated. Data are reported in the following order: chemical shift in ppm, multiplicities (br (broadened), s (singlet), d (doublet), t (triplet), q (quartet), sex (sextet), hex (hextet), m (multiplet), exch (exchangeable), app (apparent)), coupling constants, $J(\mathrm{~Hz})$, and integration. Infrared spectra were recorded on a Perkin-Elmer FTIR 1600 series spectrophotometer. Peaks are reported $\left(\mathrm{cm}^{-1}\right)$ with the following relative intensities: $\mathrm{s}$ (strong, 67-100\%), $\mathrm{m}$ (medium 40-67\%), and $\mathrm{w}$ (weak 20-40\%). Melting points were determined on a Stanford Research Systems EZ-Melt apparatus and are not corrected. High-resolution mass spectra were obtained using a VG-70-250S (double focusing) mass spectrometer at $70 \mathrm{eV}$. Analytical thin-layer chromatography was performed on Merck silica gel plates with F-254 indicator. For the starting materials that were not colored, visualization was accomplished by UV-light and $5 \%$ phosphomolybdic acid solution in ethanol. THF was dried over activated $4 \AA$ molecular sieves. All reactions were performed under a dry $\mathrm{N}_{2}$ atmosphere in oven- and or flame-dried glassware.

\section{STARTING MATERIALS}

8-Thiomethylbodipy $1,{ }^{[1]} \mathrm{CuTC},{ }^{[2]}$ were prepared according to the literature procedures. All of the other reactants, ligands and catalysts were commercially available.

Typical procedure (TP) for the cross-coupling of 1 with boronic acids. All of the reactions started with $0.06 \mathrm{mmol}(15.0 \mathrm{mg})$ of thiomethylbodipy 1 as limiting reagent and the other components of the reaction were added in the amounts stated in the TP. An oven-dry Schlenk tube, equipped with a stir bar, was charged with the boronic acid (3 equiv.), thiomethyl bodipy 1 ( 1 equiv.) and dry THF $(2 \mathrm{~mL})$ under $\mathrm{N}_{2}$. The stirred solution was sparged with $\mathrm{N}_{2}$ for 10 min., whereupon CuTC (3 equiv.), $\operatorname{Pd}_{2}(\mathrm{dba})_{3}(2.5$ mol\%), and TFP $(7.5 \mathrm{~mol} \%)$ were added under $\mathrm{N}_{2}$. The reaction mixture was immersed into a pre-heated oil bath at $55{ }^{\circ} \mathrm{C}$. After TLC showed that the reaction went to completion, the reaction mixture was allowed to reach $\mathrm{rt}$ and was adsorbed on $\mathrm{SiO}_{2}$-gel. After flash-chromatography $\left(\mathrm{SiO}_{2}\right.$-gel, EtOAc/hexanes gradient) purification, mesosubstituted bodipy's were obtained as highly-colored crystalline solids. For purposes of characterization, the products were crystallized from $\mathrm{CH}_{2} \mathrm{Cl}_{2}$ /petroleum ether.

BODIPY 2: According to TP. Orange crystals; yield=91\%; ${ }^{1} \mathrm{H}$ NMR $(200 \mathrm{MHz}$, $\left.\mathrm{CDCl}_{3}\right): \delta 7.95(\mathrm{~s}, 2 \mathrm{H}), 7.61-7.53(\mathrm{~m}, 5 \mathrm{H}), 6.94(\mathrm{~d}, \mathrm{~J}=4.4 \mathrm{~Hz}, 2.0 \mathrm{H}), 6.55(\mathrm{~d}, \mathrm{~J}=3.8 \mathrm{~Hz}$, $2 \mathrm{H}) ;{ }^{13} \mathrm{C} \mathrm{NMR}\left(50 \mathrm{MHz}, \mathrm{CDCl}_{3}\right): \delta 147.4,144.1,135.0,133.8,131.6,130.7,130.5$, $128.4,118.5 .^{[3]}$

BODIPY 3: According to TP. TLC (30\% EtOAc/hexanes, $\left.\mathrm{R}_{f}=0.7\right)$; dark green crystals; $\mathrm{mp} 166-167{ }^{\circ} \mathrm{C}$; yield=91\%; IR $\left(\mathrm{KBr}, \mathrm{cm}^{-1}\right): 1620(\mathrm{~m}), 1600(\mathrm{~m}), 1550$ (s), 1410 (s), 1391 (s), 1265 (m), 1248 (m), 1176 (m), 1121 (s), 1083 (s), 967 (m), 949 (m); ${ }^{1} \mathrm{H}$ NMR $\left(200 \mathrm{MHz}, \mathrm{CDCl}_{3}\right): 7.87$ (s, 2H), $7.56(\mathrm{~d}, J=8.8 \mathrm{~Hz}, 2 \mathrm{H}), 7.53(\mathrm{~d}, J=14.2 \mathrm{~Hz}, 1 \mathrm{H}), 7.37$ 
(s, 2H), $7.32(\mathrm{~d}, J=12.0 \mathrm{~Hz}, 1 \mathrm{H}), 7.00(\mathrm{~d}, J=9.0 \mathrm{~Hz}, 2 \mathrm{H}), 6.56(\mathrm{~m}, 2 \mathrm{H}) ; \delta^{13} \mathrm{C}(50 \mathrm{MHz}$, $\left.\mathrm{CDCl}_{3}\right): \delta 162.0,145.2,144.5,142.5,133.9,130.1,128.7,128.0,119.1,117.7,114.9$, 55.7; HRMS $\left(\mathrm{C}_{18} \mathrm{H}_{15} \mathrm{BF}_{2} \mathrm{~N}_{2} \mathrm{O}\right)$ calcd. 324.1245 found 324.1247

BODIPY 4: According to TP. TLC (30\% EtOAc/hexanes, $\left.\mathrm{R}_{f}=0.5\right)$; dark red crystals; mp 137- $138^{\circ} \mathrm{C}$; yield $=66 \%$; IR (KBr, cm $\left.{ }^{-1}\right): 1636(\mathrm{~s}), 1546(\mathrm{~s}), 1412(\mathrm{~s}), 1392(\mathrm{~s}), 1260$ (m), 1205 (m), $1120(\mathrm{~s}), 1073$ (s), 962 (m), 775 (m), 429 (m); ${ }^{1} \mathrm{H}$ NMR (200 MHz, $\left.\mathrm{CDCl}_{3}\right): \delta 7.86(\mathrm{~s}, 2 \mathrm{H}), 7.27-7.25(\mathrm{~m}, 2 \mathrm{H}), 6.85-6.61(\mathrm{~m}, 2 \mathrm{H}), 6.53(\mathrm{~m}, 2 \mathrm{H}), 2.09(\mathrm{~d}, J=$ $5.4 \mathrm{~Hz}, 3 \mathrm{H}) ;{ }^{13} \mathrm{C}$ NMR $\left(50 \mathrm{MHz}, \mathrm{CDCl}_{3}\right): \delta 144.3,144.1,143.2,133.9,128.8,124.8$, 117.9, 20.2; HRMS $\left(\mathrm{C}_{12} \mathrm{H}_{11} \mathrm{BF}_{2} \mathrm{~N}_{2}\right)$ calcd. 232.0983 found 232.0986

BODIPY 5: According to TP. TLC (30\% EtOAc/hexanes, $\left.\mathrm{R}_{f}=0.4\right)$; dark orange crystals; $\mathrm{mp} 151-152{ }^{\circ} \mathrm{C}$; yield $=71 \%$; IR $\left(\mathrm{KBr}, \mathrm{cm}^{-1}\right): 1547(\mathrm{~s}), 151(\mathrm{~m}), 1410(\mathrm{~s}), 1387$ (s), 1262 (s), 1117 (s), 1081 (s), 996 (m), 740 (m); ${ }^{1} \mathrm{H}$ NMR (200 MHz, CDCl $): \delta 7.93$ (s, 2H), 7.23-7.13 (m, 2H), 7.03-7.00 (m, 3H), $6.57(\mathrm{~m}, 2 \mathrm{H}), 3.99(\mathrm{~s}, 3 \mathrm{H}), 3.93(\mathrm{~s}, 3 \mathrm{H})$; ${ }^{13} \mathrm{C}$ NMR $\left(50 \mathrm{MHz}, \mathrm{CDCl}_{3}\right): \delta 151.8,149.0,147.6,143.7,135.0,131.5,126.7,124.4$, 118.6, 114.0, 111.1, 56.3; HRMS $\left(\mathrm{C}_{17} \mathrm{H}_{15} \mathrm{BF}_{2} \mathrm{~N}_{2} \mathrm{O}_{2}\right)$ calcd. 328.1194 found 328.1191

BODIPY 6: According to TP. TLC ( $30 \%$ EtOAc/hexanes, $\left.\mathrm{R}_{f}=0.5\right)$; orange-red crystals; mp 212-213 ${ }^{\circ} \mathrm{C}$; yield $=79 \%$; IR $\left(\mathrm{KBr}, \mathrm{cm}^{-1}\right) 3115(\mathrm{w}), 1567(\mathrm{~s}), 1543(\mathrm{~m}), 1413(\mathrm{~s})$, 1390(s), 1265(m), 1161(m), $1126(\mathrm{~s}), 1080(\mathrm{~s}), 1070(\mathrm{~s}), 981(\mathrm{~m}), 913(\mathrm{~m}), 775(\mathrm{~m}), 751$ (m), $741(\mathrm{~m}), 701(\mathrm{w}) .{ }^{1} \mathrm{H}$ NMR $\left(200 \mathrm{MHz}, \mathrm{CDCl}_{3}\right) \delta 7.96(\mathrm{~s}, 2 \mathrm{H}), 7.78-7.639(\mathrm{~m}, 6 \mathrm{H})$, 7.55-7.425 (m, 3H), $7.03(\mathrm{~d}, J=4 \mathrm{~Hz}, 2 \mathrm{H}), 6.57(\mathrm{~d}, J=2.8 \mathrm{~Hz}, 2 \mathrm{H}) ;{ }^{13} \mathrm{C}$ NMR $(50 \mathrm{MHz}$, $\mathrm{CDCl}_{3}$ ): 144.3, 144.0, 140.0, 135.1, 132.9, 131.8, 131.4, 129.3, 128.4, 127.4, 127.3, 118.8; HMRS $\left(\mathrm{C}_{21} \mathrm{H}_{15} \mathrm{BF}_{2} \mathrm{~N}_{2}\right)$ : calcd, 344.1296 found 344.1303.

BODIPY 7: According to TP. TLC (30\% EtOAc/hexanes, $\left.\mathrm{R}_{f}=0.6\right)$; orange crystals; $\mathrm{mp}$ 202-203 ${ }^{\circ} \mathrm{C}$; yield $=81 \%$; IR $\left(\mathrm{KBr}, \mathrm{cm}^{-1}\right) 1566(\mathrm{~m}), 1548(\mathrm{~s}), 1410(\mathrm{~m}), 1387(\mathrm{~s}), 1258$ (m), $1110(\mathrm{~s}), 1075(\mathrm{~s}), 978(\mathrm{~m}){ }^{1} \mathrm{H}$ NMR $\left(200 \mathrm{MHz}, \mathrm{CDCl}_{3}\right) \delta 7.79(\mathrm{~s}, 2 \mathrm{H}), 7.61-7.58$ (m, 2H), 7.49-7.46 (m, 2H), 7.25-7.17 (m, 5H), $6.72(\mathrm{~d}, J=4.2 \mathrm{~Hz}, 2 \mathrm{H}), 6.38$ (d, $J=3.1$ $\mathrm{Hz}, 2 \mathrm{H}) ;{ }^{13} \mathrm{C}$ NMR $\left(50 \mathrm{MHz}, \mathrm{CDCl}_{3}\right) \delta 147.3,144.0,142.1,140.2,135.8,132.4,131.5$, 130.9, 130.5, 128.9, 128.6, 127.7, 127.0, 118.5; HRMS $\left(\mathrm{C}_{21} \mathrm{H}_{15} \mathrm{BF}_{2} \mathrm{~N}_{2}\right)$ : calcd. 344.1296 found 344.1298. Anal. Calcd for $\mathrm{C}_{21} \mathrm{H}_{15} \mathrm{BF}_{2} \mathrm{~N}_{2}$ : C, 73.29; H, 4.39; N, 8.14. Found: $\mathrm{C}$, 73.36; H, 4.35; N, 7.99 .

BODIPY 8: According to TP. TLC ( $30 \%$ EtOAc/hexanes, $\left.\mathrm{R}_{f}=0.4\right)$; orange crystals; $\mathrm{mp}$ 170-172 ${ }^{\circ} \mathrm{C}$; yield $=96 \%$; IR $\left(\mathrm{KBr}, \mathrm{cm}^{-1}\right) 3136(\mathrm{w}), 1564(\mathrm{~s}), 1539(\mathrm{~s}), 1414(\mathrm{~s}), 1386(\mathrm{~s})$, 1261 (s), 1115 (s), 1081 (s), 977 (m); ${ }^{1} \mathrm{H}$ NMR (200 MHz, CDCl $) \delta 7.94(\mathrm{~s}, 2 \mathrm{H}), 7.55$ (s, $4 \mathrm{H}), 6.96(\mathrm{~d}, J=4.0 \mathrm{~Hz}, 2 \mathrm{H}), 6.81\left(\mathrm{dd}, J_{1}=18.0 \mathrm{~Hz}, J_{2}=10.0 \mathrm{~Hz}, 1 \mathrm{H}\right), 6.56-6.54(\mathrm{~m}$, $2 \mathrm{H}), 5.91\left(\mathrm{dd}, J_{1}=18.0 \mathrm{~Hz}, J_{2}=0.8 \mathrm{~Hz}, 1 \mathrm{H}\right), 5.43\left(\mathrm{dd}, J_{1}=10.0 \mathrm{~Hz}, J_{2}=0.8 \mathrm{~Hz}, 1 \mathrm{H}\right)$; ${ }^{13} \mathrm{C}$ NMR $\left(50 \mathrm{MHz}, \mathrm{CDCl}_{3}\right) \delta 147.3,144.2,140.4,136.0,135.0,133.3,131.6,131.1$, 126.4, 118.7, 116.5; HRMS $\left(\mathrm{C}_{17} \mathrm{H}_{13} \mathrm{BF}_{2} \mathrm{~N}_{2}\right)$ : calcd. 294.1139 found 294.1140. 
BODIPY 9: According to TP. TLC (30\% EtOAc/hexanes, $\left.\mathrm{R}_{f}=0.25\right)$; dark orange crystals; mp 177-178 ${ }^{\circ} \mathrm{C}$; yield= 83\%; IR $\left(\mathrm{KBr}, \mathrm{cm}^{-1}\right) 3109$ (w), $1590(\mathrm{~m}), 1563$ (s), 1537 (s), 1477 (m), 1412 (s), 1385 (s), 1261 (s), 1157 (m), 1117 (s), 1079 (s), 1047 (m), 976 (m), 910 (m), $774(\mathrm{~m}), 740(\mathrm{~m}) ;{ }^{1} \mathrm{H}$ NMR $\left(200 \mathrm{MHz}, \mathrm{CDCl}_{3}\right) \delta 7.94$ (s, 2H), 7.52 (d, J= $8.0 \mathrm{~Hz}, 2 \mathrm{H}), 7.37$ (d, $J=8.0 \mathrm{~Hz}, 2 \mathrm{H}), 6.98(\mathrm{~d}, J=4.0 \mathrm{~Hz}, 2 \mathrm{H}), 6.56(\mathrm{~d}, J=4.0 \mathrm{~Hz}, 2 \mathrm{H})$, $3.58(\mathrm{~s}, 3 \mathrm{H}) ;{ }^{13} \mathrm{C} \mathrm{NMR}\left(50 \mathrm{MHz}, \mathrm{CDCl}_{3}\right) \delta 144.0,143.5,133.0,131.5,131.2,130.0$, 125.6, 121.9, 118.8, 15.3; HMRS $\left(\mathrm{C}_{16} \mathrm{H}_{13} \mathrm{BF}_{2} \mathrm{~N}_{2} \mathrm{~S}\right)$ : Calcd, 314.0860 found 314.0855.

BODIPY 10: According to TP. TLC (30\% EtOAc/hexanes, $\left.\mathrm{R}_{f}=0.3\right)$; dark red crystals; mp 233-235 ${ }^{\circ} \mathrm{C}$; yield= 76\%; IR (KBr, cm $\left.{ }^{-1}\right) 3116(\mathrm{w}), 2922(\mathrm{w}), 2841(\mathrm{w}), 1699(\mathrm{~s})$, 1574 (s), 1547 (s), 1413 (s), 1390 (s), 1263 (s), 1157 (m), 1120 (s), 1088 (s), 1045 (m), $975(\mathrm{~m}) ;{ }^{1} \mathrm{H}$ NMR $\left(200 \mathrm{MHz}, \mathrm{CDCl}_{3}\right) \delta 10.16(\mathrm{~s}, 1 \mathrm{H}), 8.06(\mathrm{~d}, J=8.4 \mathrm{~Hz}, 2 \mathrm{H}), 7.99(\mathrm{~s}$, 2H), $7.51(\mathrm{~d}, J=8.4 \mathrm{~Hz}, 2 \mathrm{H}), 6.88(\mathrm{~d}, J=4.4,2 \mathrm{H}), 6.58(\mathrm{~d}, J=4.0,2 \mathrm{H}) ;{ }^{13} \mathrm{C}$ NMR $(50$ $\left.\mathrm{MHz} \mathrm{CDCl}_{3}\right) \delta 191.5,145.3,139.6,137.8,134.8,131.6,131.2,129.7,119.3$; HRMS $\left(\mathrm{C}_{16} \mathrm{H}_{11} \mathrm{BF}_{2} \mathrm{~N}_{2} \mathrm{O}\right)$ calcd. 296.0932 found 296.0937.

BODIPY 11: According to TP. TLC (30\% EtOAc/hexanes, $\left.\mathrm{R}_{f}=0.5\right)$; bright green crystals; $\mathrm{mp}>195{ }^{\circ} \mathrm{C}$ (dec.); yield $=78 \%$; IR $\left(\mathrm{KBr}, \mathrm{cm}^{-1}\right) 3107(\mathrm{w}), 2912(\mathrm{w}), 2812(\mathrm{w})$, 1598 (s), 1551 (s), 1527 (s), 1409 (s), 1389 (s), 1372 (s), 1263 (s), 1199 (s), 1120 (s), 1079 (s), 1043 (m), 975 (s); ${ }^{1} \mathrm{H}$ NMR $\left(200 \mathrm{MHz}, \mathrm{CDCl}_{3}\right) \delta 7.87$ (s, 2H), 7.56 (d, J=9.0 $\mathrm{Hz}, 2 \mathrm{H}), 7.04(\mathrm{~d}, J=4.2,2 \mathrm{H}), 6.81(\mathrm{~d}, J=9.0 \mathrm{~Hz}, 2 \mathrm{H}), 6.56-6.54(\mathrm{~m}, 2 \mathrm{H}), 3.12(\mathrm{~s}, 6 \mathrm{H})$; ${ }^{13} \mathrm{C}$ NMR $\left(50 \mathrm{MHz}, \mathrm{CDCl}_{3}\right) \delta 152.7,148.6,141.9,134.7,133.3,131.0,122.2,117.8$, 111.8, 40.4; HRMS $\left(\mathrm{C}_{17} \mathrm{H}_{16} \mathrm{BF}_{2} \mathrm{~N}_{3}\right)$ : calcd. 311.1405, found 311.1408.

BODIPY 12: According to TP. TLC (30\% EtOAc/hexanes, $\left.\mathrm{R}_{f}=0.5\right)$; orange crystals; mp 202-203 ${ }^{\circ} \mathrm{C}$; yield = 78\%; IR (KBr, cm $\left.{ }^{-1}\right) 3133$ (w), 3109 (w), 1569 (s), 1541 (s), 1414 (s), 1398 (s), 1384 (s), 1260 (s), 1155 (m), 1117 (s), 1082 (s), 1048 (m), 977 (m); ${ }^{1} \mathrm{H}$ $\operatorname{NMR}\left(200 \mathrm{MHz}, \mathrm{CDCl}_{3}\right) \delta 7.96(\mathrm{~s}, 2 \mathrm{H}), 7.69\left(\mathrm{dd}, J_{I}=6.6 \mathrm{~Hz}, J_{2}=2.0,2 \mathrm{H}\right), 7.45\left(\mathrm{dd}, J_{I}=\right.$ $\left.6.8 \mathrm{~Hz}, J_{2}=2.0,2 \mathrm{H}\right), 6.91(\mathrm{~d}, J=4.0,2 \mathrm{H}), 6.57(\mathrm{~d}, J=3.8,2 \mathrm{H}) ;{ }^{13} \mathrm{C} \mathrm{NMR}(50 \mathrm{MHz}$, $\left.\mathrm{CDCl}_{3}\right) \delta 1$ 146.0, 144.8, 134.9, 132.8, 132.0, 131.5, 125.7, 119.0; HRMS $\left(\mathrm{C}_{15} \mathrm{H}_{10} \mathrm{BBrF}_{2} \mathrm{~N}_{2}\right)$ : calcd. 346.0088 found 346.0088 .

BODIPY 13: According to TP. TLC (40\% EtOAc/hexanes, $\left.\mathrm{R}_{f}=0.7\right)$; orange crystals; $\mathrm{mp} 135^{\circ} \mathrm{C}$; yield = 62\%; IR (KBr, cm $\left.{ }^{-1}\right) 1564(\mathrm{~m}), 1543(\mathrm{~s}), 1414(\mathrm{~m}), 1385$ (s), 1079 (s), $985(\mathrm{~m}) ;{ }^{1} \mathrm{H}$ NMR $\left(200 \mathrm{MHz}, \mathrm{CDCl}_{3}\right) \delta 7.96(\mathrm{~s}, 2 \mathrm{H}), 7.75-7.71(\mathrm{~m}, 2 \mathrm{H}), 7.52-7.37(\mathrm{~m}$, $2 \mathrm{H}), 6.92(\mathrm{~d}, J=4.4 \mathrm{~Hz}, 2 \mathrm{H}), 6.57(\mathrm{~d}, J=3.8 \mathrm{~Hz}, 2 \mathrm{H}) ;{ }^{13} \mathrm{C} \mathrm{NMR}\left(50 \mathrm{MHz}, \mathrm{CDCl}_{3}\right) \delta$ 145.3, 145.0, 135.8, 135.0, 133.9, 133.1, 131.6, 130.2, 129.2, 122.8, 119.1; HRMS $\left(\mathrm{C}_{15} \mathrm{H}_{10} \mathrm{BBrF}_{2} \mathrm{~N}_{2}\right)$ : calcd. 346.0088 found 346.0094 .

BODIPY 14: According to TP. TLC (20\% EtOAc/hexanes, $\left.\mathrm{R}_{f}=0.3\right)$; orange crystals; mp 150-152 ${ }^{\circ} \mathrm{C}$; yield= 83\%; IR (KBr, cm $\left.{ }^{-1}\right) 3111(\mathrm{w}), 2929(\mathrm{w}), 1569(\mathrm{~s}), 1411(\mathrm{~m})$, 1387 (s), 1257 (s), 1108 (s), 1068 (s), 938 (m), 741 (m); ${ }^{1} \mathrm{H}$ NMR (200 MHz, CDCl $\left.\mathrm{C}_{3}\right) \delta$ $7.95(\mathrm{~s}, 2 \mathrm{H}), 7.76-7.71(\mathrm{~m}, 1 \mathrm{H}), 7.47-7.37(\mathrm{~m}, 3 \mathrm{H}), 6.72(\mathrm{~d}, J=4.0,2 \mathrm{H}), 6.51(\mathrm{~d}, J=4.4$, 
$2 \mathrm{H}) ;{ }^{13} \mathrm{C}$ NMR $\left(50 \mathrm{MHz}, \mathrm{CDCl}_{3}\right) \delta 145.2,145.0,135.4,134.6,133.5,131.4,131.3$, 131.2, 127.2, 122.8, 119.0; HRMS $\left(\mathrm{C}_{15} \mathrm{H}_{10} \mathrm{BBrF}_{2} \mathrm{~N}_{2}\right)$ : calcd. 346.0088 found 346.0096.

BODIPY 15: According to TP. TLC (20\% EtOAc/hexanes, $\left.\mathrm{R}_{f}=0.4\right)$; green-orange crystals; $\mathrm{mp}>240{ }^{\circ} \mathrm{C}$; yield $=67 \%$; IR $\left(\mathrm{KBr}, \mathrm{cm}^{-1}\right) 3111(\mathrm{w}), 3036(\mathrm{w}), 2926(\mathrm{w}), 1557$ (s), 1412 (m), 1385 (s), 1257 (m), 1108 (s), 1071 (s), 1038 (m), 985 (m); ${ }^{1} \mathrm{H}$ NMR (200 $\left.\mathrm{MHz}_{\mathrm{CDCl}}\right) \delta 8.31-8.01(\mathrm{~m}, 11 \mathrm{H}), 6.64(\mathrm{~d}, J=4.2,2 \mathrm{H}), 6.48(\mathrm{~d}, J=3.0,2 \mathrm{H}) ;{ }^{13} \mathrm{C}$ NMR $\left(50 \mathrm{MHz}, \mathrm{CDCl}_{3}\right) \delta 146.5,144.4,136.5,132.5,131.7,131.2,130.7,130.4,129.0$, $128.5,127.8,127.4,127.1,126.6,126.1,125.9,124.9,124.5,124.2,124.0,118.7$; HRMS $\left(\mathrm{C}_{25} \mathrm{H}_{15} \mathrm{BF}_{2} \mathrm{~N}_{2}\right)$ : calcd. 392.1296 found 392.1303 .

BODIPY 16: According to TP. TLC (20\% EtOAc/hexanes, $\left.\mathrm{R}_{f}=0.2\right)$; green-purple crystals; $\mathrm{mp}>220{ }^{\circ} \mathrm{C}$ (dec.); yield $=98 \%$; IR ( KBr, cm $\left.{ }^{-1}\right) 3112(\mathrm{w}), 1529$ (s), 11411 (s), 1385 (s), 1265 (s), 1124 (s), 1082 (s), 978 (m); ${ }^{1} \mathrm{H}$ NMR (200 MHz, CDCl $\left.{ }_{3}\right) \delta 7.86$ (s, $2 \mathrm{H}), 7.66(\mathrm{~d}, J=4.2 \mathrm{~Hz}, 2 \mathrm{H}), 6.55-6.53(\mathrm{~m}, 2 \mathrm{H}), 4.98(\mathrm{t}, J=2.0 \mathrm{~Hz}, 2 \mathrm{H}), 4.76(\mathrm{t}, J=2.0$ $\mathrm{Hz}, 2 \mathrm{H}), 4.22(\mathrm{~s}, 5 \mathrm{H}) ;{ }^{13} \mathrm{C} \mathrm{NMR}\left(50 \mathrm{MHz}, \mathrm{CDCl}_{3}\right) \delta 151.8,141.0,135.0,130.2,117.6$, 79.4, 74.3, 72.3, 72.1; HRMS $\left(\mathrm{C}_{19} \mathrm{H}_{15} \mathrm{BF}_{2} \mathrm{FeN}_{2}\right)$ : calcd. 376.0645 found 376.0647 .

BODIPY 17: According to TP. TLC (20\% EtOAc/hexanes, $\left.\mathrm{R}_{f}=0.5\right)$; bright green crystals; mp 219-220 ${ }^{\circ} \mathrm{C}$; yield $=93 \%$; IR $\left(\mathrm{KBr}, \mathrm{cm}^{-1}\right) 1551$ (s), 1451 (w), $1413(\mathrm{~m}), 1386$ (s), 1355 (m), 1257 (s), 1187 (m), 1109 (s), 1073 (s), 987 (w), 937 (w), 754 (m); ${ }^{1} \mathrm{H} \mathrm{NMR}$ $\left(200 \mathrm{MHz}, \mathrm{CDCl}_{3}\right) \delta 8.16\left(\mathrm{dd}, J_{l}=7.4, J_{2}=1.6 \mathrm{~Hz}, 1 \mathrm{H}\right), 8.05-7.99(\mathrm{~m}, 3 \mathrm{H}), 7.60-7.37(\mathrm{~m}$, $5 \mathrm{H}), 6.88(\mathrm{~d}, J=4.0 \mathrm{~Hz}, 2 \mathrm{H}), 6.52(\mathrm{~d}, J=3.4 \mathrm{~Hz}, 2 \mathrm{H}) ;{ }^{13} \mathrm{C} \mathrm{NMR}\left(50 \mathrm{MHz}, \mathrm{CDCl}_{3}\right) \delta$ 156.3, 144.8, 135.5, 131.8, 129.4, 128.3, 125.5, 123.7, 123.6, 123.1, 122.8, 121.0, 118.8, 118.3, 112.4; HMRS $\left(\mathrm{C}_{21} \mathrm{H}_{13} \mathrm{BF}_{2} \mathrm{~N}_{2} \mathrm{O}\right)$ : calcd. 358.1089 found 358.1080.

BODIPY 18: According to TP. TLC (25\% EtOAc/hexanes, $\left.\mathrm{R}_{f}=0.35\right)$; orange crystals; $\mathrm{mp} 137-138^{\circ} \mathrm{C}$; yield $=81 \%$; IR $\left(\mathrm{KBr}, \mathrm{cm}^{-1}\right): 3015(\mathrm{~m}), 1602$ (s), 1542 (s), 1411 (s), 1388 (s), 1259 (s), 1120 (s), 1077 (s). ${ }^{1} \mathrm{H}$ NMR (200 MHz, CDCl 3 ) $\delta 7.92$ (s, 2H), 7.55 (dt, $J_{I}=$ $\left.8.8 \mathrm{~Hz}, J_{2}=2.1 \mathrm{~Hz}, 2 \mathrm{H}\right), 7.05\left(\mathrm{dt}, J_{l}=9.0 \mathrm{~Hz}, J_{2}=2.2 \mathrm{~Hz}, 2 \mathrm{H}\right), 6.98(\mathrm{~d}, J=4.0 \mathrm{~Hz}, 2 \mathrm{H})$, 6.56-6.54 (m, 2H), $3.92(\mathrm{~s}, 3 \mathrm{H}) ;{ }^{13} \mathrm{C} \mathrm{NMR}\left(50 \mathrm{MHz}, \mathrm{CDCl}_{3}\right): \delta 162.3,147.7,143.6$, 135.1, 132.6, 131.6, 126.6, 118.4, 114.3, 55.7; HRMS $\left(\mathrm{C}_{16} \mathrm{H}_{13} \mathrm{BF}_{2} \mathrm{~N}_{2} \mathrm{O}\right)$ : calcd. 298.1089 found 298.1090 .

BODIPY 19: According to TP. TLC (20\% EtOAc/hexanes, $\left.\mathrm{R}_{f}=0.3\right)$; orange crystals; mp 90-92 ${ }^{\circ} \mathrm{C}$; yield = 78\%; IR (KBr, cm $\left.{ }^{-1}\right) 3106$ (w), 1540 (s), 1408 (s), 1386 (s), 1263 (s), 1120 (s), 1085 (s), 969 (s); $\left.{ }^{1} \mathrm{H} \mathrm{NMR} \mathrm{(200} \mathrm{MHz,} \mathrm{CDCl}{ }_{3}\right) \delta 7.93$ (s, 2H), 7.72 (dd, $J_{1}=$ $\left.5.0 \mathrm{~Hz}, J_{2}=1.2 \mathrm{~Hz}, 1 \mathrm{H}\right), 7.58\left(\mathrm{dd}, J_{1}=3.8 \mathrm{~Hz}, J_{2}=1.2 \mathrm{~Hz}, 1 \mathrm{H}\right), 7.30-7.27(\mathrm{~m}, 3 \mathrm{H}), 6.58$ $(\mathrm{d}, J=3.6,2 \mathrm{H}) ;{ }^{13} \mathrm{C} \mathrm{NMR}\left(50 \mathrm{MHz}, \mathrm{CDCl}_{3}\right) \delta 144.1,139.8,134.7,134.5,133.2,131.7$, 128.5, 118.7; HRMS $\left(\mathrm{C}_{13} \mathrm{H}_{9} \mathrm{BF}_{2} \mathrm{~N}_{2} \mathrm{~S}\right)$ : calcd. 274.0547 found 274.0546.

BODIPY 20: According to TP. TLC (20\% EtOAc/hexanes, $\left.\mathrm{R}_{f}=0.3\right)$; green-orange crystals; mp 116-118 ${ }^{\circ} \mathrm{C}$; yield = 84\%; IR $\left(\mathrm{KBr}, \mathrm{cm}^{-1}\right) 3121(\mathrm{w}), 2920(\mathrm{w}), 1542$ (s), 1410 (s), 1385 (s), 1258 (s), 1115 (s), 1072 (s), 968 (m); ${ }^{1} \mathrm{H}$ NMR (200 MHz, CDCl $\left.{ }_{3}\right) \delta 7.90$ (s, 2H), 7.42 (d, $J=3.6 \mathrm{~Hz}, 1 \mathrm{H}), 7.32$ (d, $J=4.2 \mathrm{~Hz}, 2 \mathrm{H}), 6.96-6.95(\mathrm{~m}, 1 \mathrm{H}), 6.57$ (d, $J=$ 
$3.4 \mathrm{~Hz}, 2 \mathrm{H}), 2.63(\mathrm{~s}, 3 \mathrm{H}) ;{ }^{13} \mathrm{C} \mathrm{NMR}\left(50 \mathrm{MHz}, \mathrm{CDCl}_{3}\right) \delta 148.0,143.4,140.2,134.3$, 134.1, 132.6, 131.5, 127.3, 118.4, 15.9; HRMS $\left(\mathrm{C}_{14} \mathrm{H}_{11} \mathrm{BF}_{2} \mathrm{~N}_{2} \mathrm{~S}\right)$ : calcd. 288.0704 found 288.0704 .

BODIPY 21: According to TP. TLC (40\% EtOAc/hexanes, $\left.\mathrm{R}_{f}=0.5\right)$; dark red crystals; mp 132-134 ${ }^{\circ} \mathrm{C}$; yield $=87 \%$; IR $\left(\mathrm{KBr}, \mathrm{cm}^{-1}\right) 3125(\mathrm{w}), 1574(\mathrm{~m}), 1540$ (s), 1412 (s), 1387 (s), 1077 (s), 986 (s); ${ }^{1} \mathrm{H}$ NMR (200 MHz, $\left.\mathrm{CDCl}_{3}\right) \delta 7.89$ (s, 2H), $7.84(\mathrm{~d}, J=1.7 \mathrm{~Hz}, 1 \mathrm{H})$, $7.47(\mathrm{~d}, J=4.0 \mathrm{~Hz}, 2 \mathrm{H}), 7.21(\mathrm{~d}, J=3.6 \mathrm{~Hz}, 1 \mathrm{H}), 6.72\left(\mathrm{dd}, J_{l}=3.5 \mathrm{~Hz}, J_{2}=1.8 \mathrm{~Hz}, 1 \mathrm{H}\right)$, $6.59(\mathrm{~d}, J=3.1 \mathrm{~Hz}, 2 \mathrm{H}) ;{ }^{13} \mathrm{C}$ NMR $\left(50 \mathrm{MHz}, \mathrm{CDCl}_{3}\right) \delta 148.8,148.0,143.3,133.0,132.5$, 130.9, 120.8, 118.5, 113.6; HRMS $\left(\mathrm{C}_{13} \mathrm{H}_{9} \mathrm{BF}_{2} \mathrm{~N}_{2} \mathrm{O}\right)$ : calcd. 258.0776 found 258.0780.

Metathesis reaction of 8 . A two-necked oven-dry round-bottomed flask was equipped with a stir-bar, a condenser, a $\mathrm{N}_{2}$ inlet, and a rubber septum. The system was charged with bodipy 9 (20 mg, $0.07 \mathrm{mmol})$, benzylidene[1,3-bis(2,4,6-trimethylphenyl)-2imidazolidinylidene]dichoso(tricyclohexylphosphine)ruthenium (Grubbs catalys, 2nd. generation) $(5.77 \mathrm{mg}, 0.007 \mathrm{mmol})$, and dry $\mathrm{CH}_{2} \mathrm{Cl}_{2}$. The reaction mixture was refluxed for $1.5 \mathrm{~h}$, cooled down to $\mathrm{rt}$ and the solvent was removed in vacuo. The product was purified by gravity column chromatography $\left(\mathrm{SiO}_{2}\right.$-gel, AcOEt/hexanes gradient) to give 22 as a brown crystalline solid (15.4 mg, 81\%). TLC (20\% EtOAc/hexanes, $\left.\mathrm{R}_{f}=0.1\right)$; $\mathrm{mp}>220^{\circ} \mathrm{C}$; IR (KBr, cm $\left.{ }^{-1}\right) 2928(\mathrm{w}), 1562(\mathrm{~m}), 1410$ (m), 1389 (s), 1263 (m), $1118(\mathrm{~s})$, $1079(\mathrm{~m}), 981(\mathrm{~m}) ;{ }^{1} \mathrm{H} \mathrm{NMR}\left(200 \mathrm{MHz}, \mathrm{CDCl}_{3}\right) \delta 7.97(\mathrm{~s}, 4 \mathrm{H}), 7.73(\mathrm{~d}, J=8.4 \mathrm{~Hz}, 4 \mathrm{H})$, $7.63(\mathrm{~d}, J=8.4 \mathrm{~Hz}, 4 \mathrm{H}), 7.34(\mathrm{~s}, 1 \mathrm{H}), 7.00(\mathrm{~d}, J=3.8 \mathrm{~Hz}, 4 \mathrm{H}), 6.58(\mathrm{~d}, J=2.6 \mathrm{~Hz}, 4 \mathrm{H})$; ${ }^{13} \mathrm{C}$ NMR $\left(50 \mathrm{MHz}, \mathrm{CDCl}_{3}\right) \delta 147.0,144.4,139.7,135.0,133.8,131.4,129.8,128.6$, 127.0, 118.8; HRMS $\left(\mathrm{C}_{32} \mathrm{H}_{22} \mathrm{~B}_{2} \mathrm{~F}_{4} \mathrm{~N}_{4}\right)$ : calcd. 560.1966 found 560.1953.

Suzuki cross-coupling reaction of 12 . An oven-dry Schlenk tube containing a stir-bar, was charged with 4-biphenylboronic acid (17.1 mg, $0.09 \mathrm{mmol}), \mathrm{Pd}\left(\mathrm{PPh}_{3}\right)_{4}(1.99 \mathrm{mg}$, $0.002 \mathrm{mmol})$, and $\mathrm{K}_{3} \mathrm{PO}_{4}(24.4 \mathrm{mg}, 0.12 \mathrm{mmol})$ whereupon a 6:1 (v:v) DME: $\mathrm{H}_{2} \mathrm{O}$ mixture $(3 \mathrm{~mL})$ was added. $\mathrm{N}_{2}$ was bubbled through the solution for $5 \mathrm{~min}$ and then bodipy 12 (20.0 $\mathrm{mg}, 0.06 \mathrm{mmol})$ was added. The reaction container was then immersed into a pre-heated oil-bath at $80{ }^{\circ} \mathrm{C}$ for $1 \mathrm{~h}$. The reaction mixture was cooled to $\mathrm{rt}$ and the solvent was removed under reduced pressure. The crude material was purified by gravity column chromatography ( $\mathrm{SiO}_{2}$-gel, AcOEt/hexanes gradient) to give $\mathbf{2 3}$ as an orangegreen crystalline solid (14.4 mg, 59\%). TLC (20\% EtOAc/hexanes, $\left.\mathrm{R}_{f}=0.4\right) ; \mathrm{mp}>220$ ${ }^{\circ} \mathrm{C}$; IR (KBr, cm $\left.{ }^{-1}\right) 3113$ (w), 3033 (w), 2919 (w), 1551 (s), 1558 (s), 1412 (s), 1387 (s), 1263 (s), 1159 (m), 1117 (s), 10079 (s), 1050 (m), 977 (m); ${ }^{1} \mathrm{H}$ NMR (200 MHz, CDCl 3 ) $\delta 7.97(\mathrm{~s}, 2 \mathrm{H}), 7.83-7.66(\mathrm{~m}, 10 \mathrm{H}), 7.52-7.39(\mathrm{~m}, 3 \mathrm{H}), 7.03(\mathrm{~d}, J=4.0 \mathrm{~Hz}, 2 \mathrm{H}), 6.58(\mathrm{~d}$, $J=2.4 \mathrm{~Hz}, 2 \mathrm{H}) ;{ }^{13} \mathrm{C}$ NMR $\left(50 \mathrm{MHz}, \mathrm{CDCl}_{3}\right) \delta 147.3,144.3,143.5,141.3,140.6,138.8$, $135.1,133.0,131.8,131.4,129.1,128.0,127.8,127.8,127.3,127.2,118.7$; HRMS $\left(\mathrm{C}_{27} \mathrm{H}_{19} \mathrm{BF}_{2} \mathrm{~N}_{2}\right)$ : calcd. 420.1609 , found 420.1602 . 


\section{References:}

[1] T. V. Goud, A. Tutar, J.-F. Biellmann, Tetrahedron 2006, 62, 5084.

[2] During the progress of this work, we noticed that shorter reaction times were observed when freshly-prepared $\mathrm{Cu}(\mathrm{I}) \mathrm{TC}$ was used: S. Zhang, D. Zhang, L. S. Liebeskind, J. Org. Chem. 1997, 62, 2312-2313. Cu(I)TC is also commercially available.

[3] R. W. Wagner, J. S. Lindsey, Pure \& Appl. Chem. 1996, 68(7), 1373. 


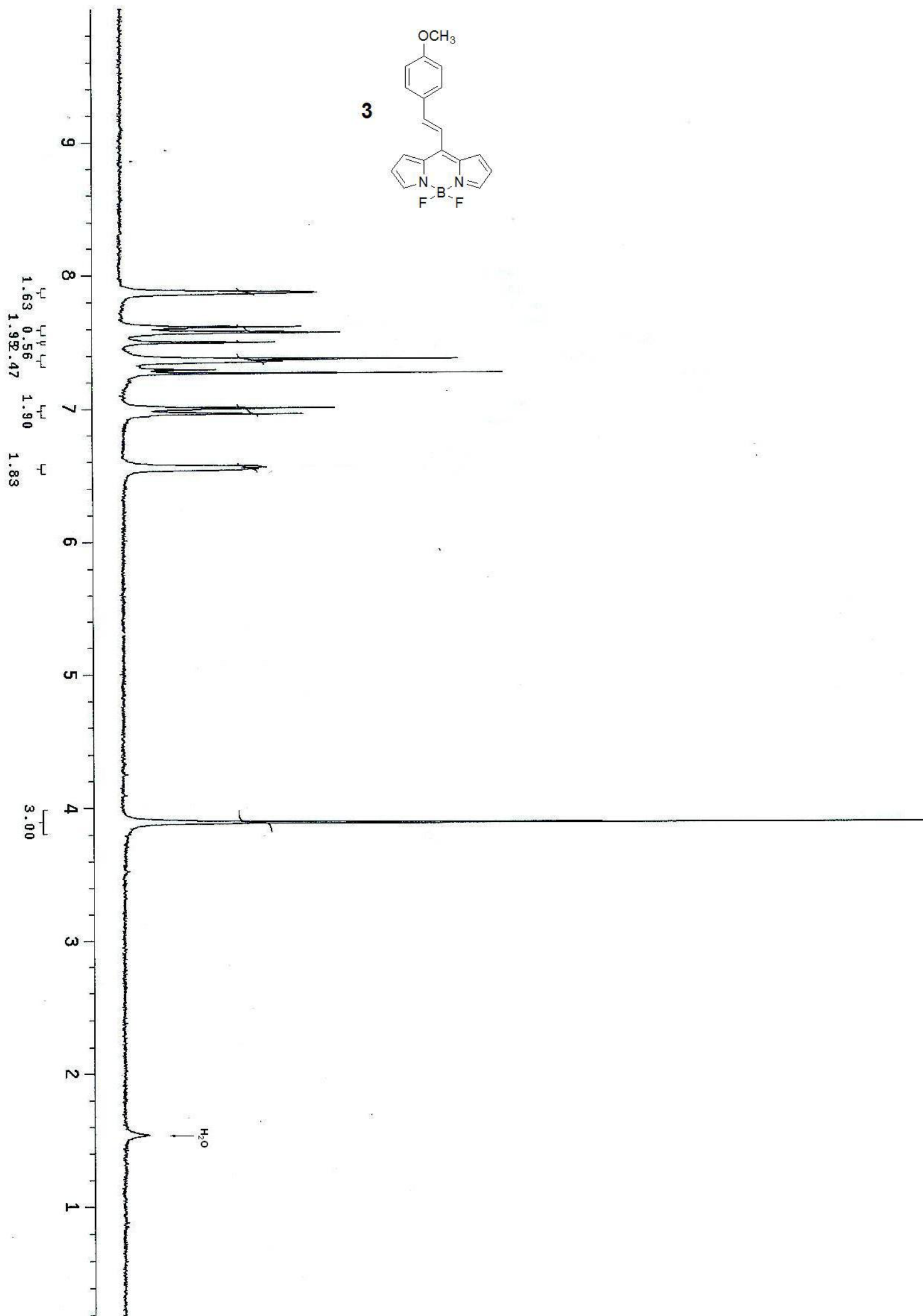




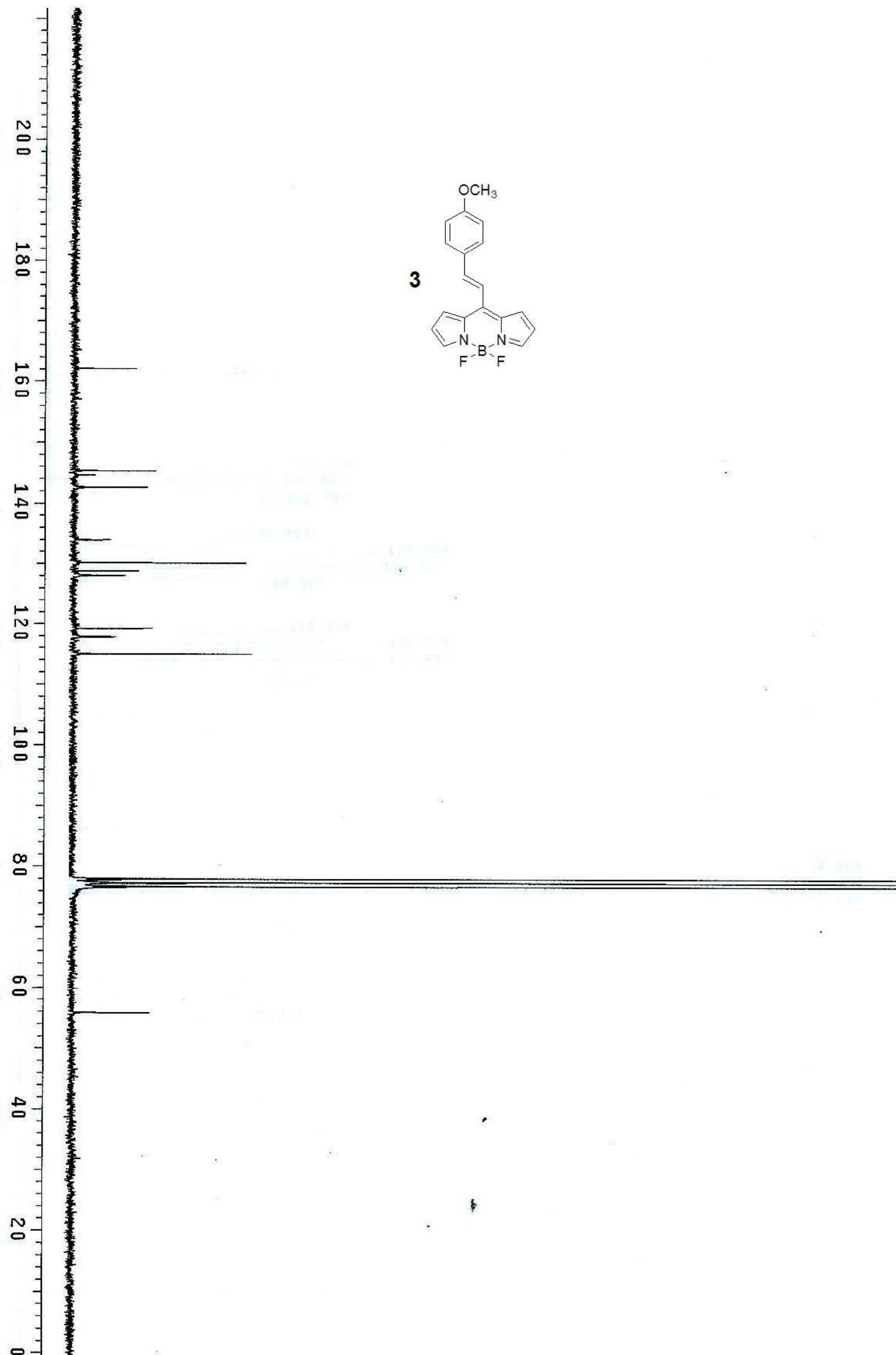


S10

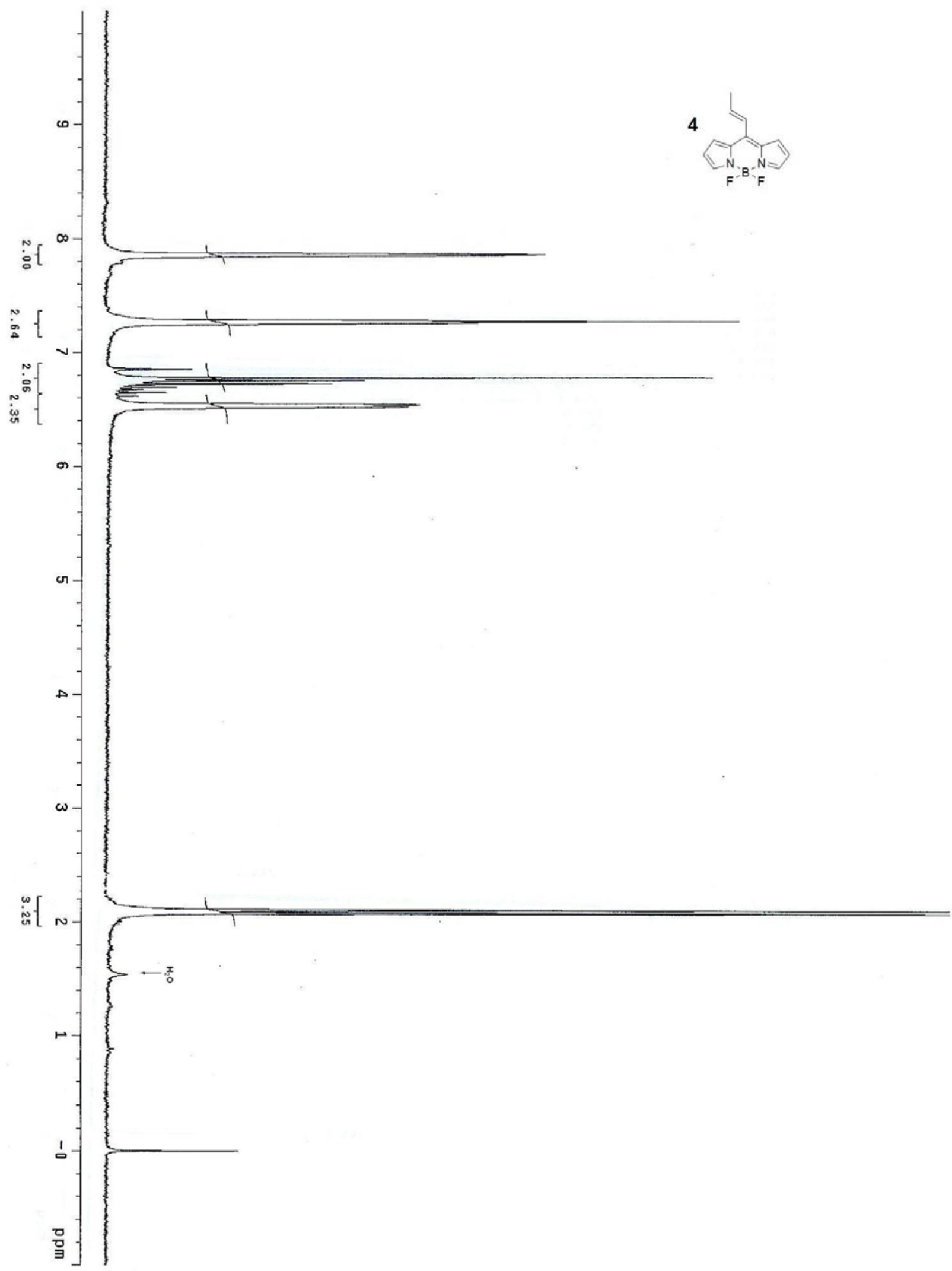


S11

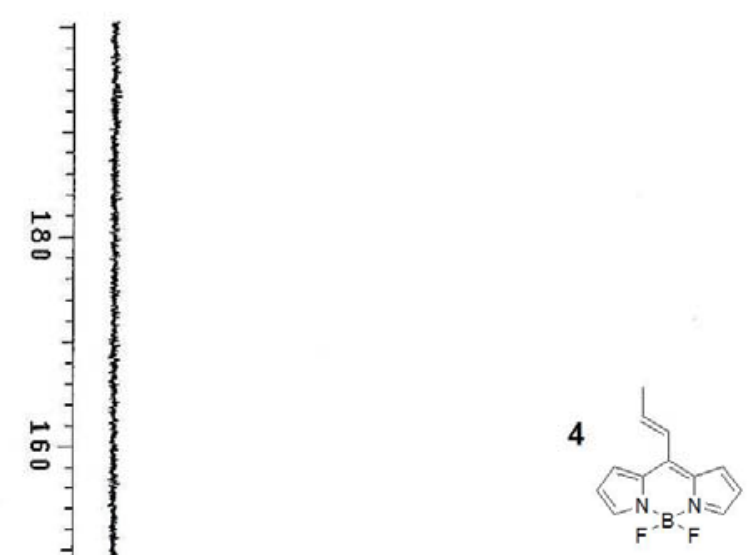




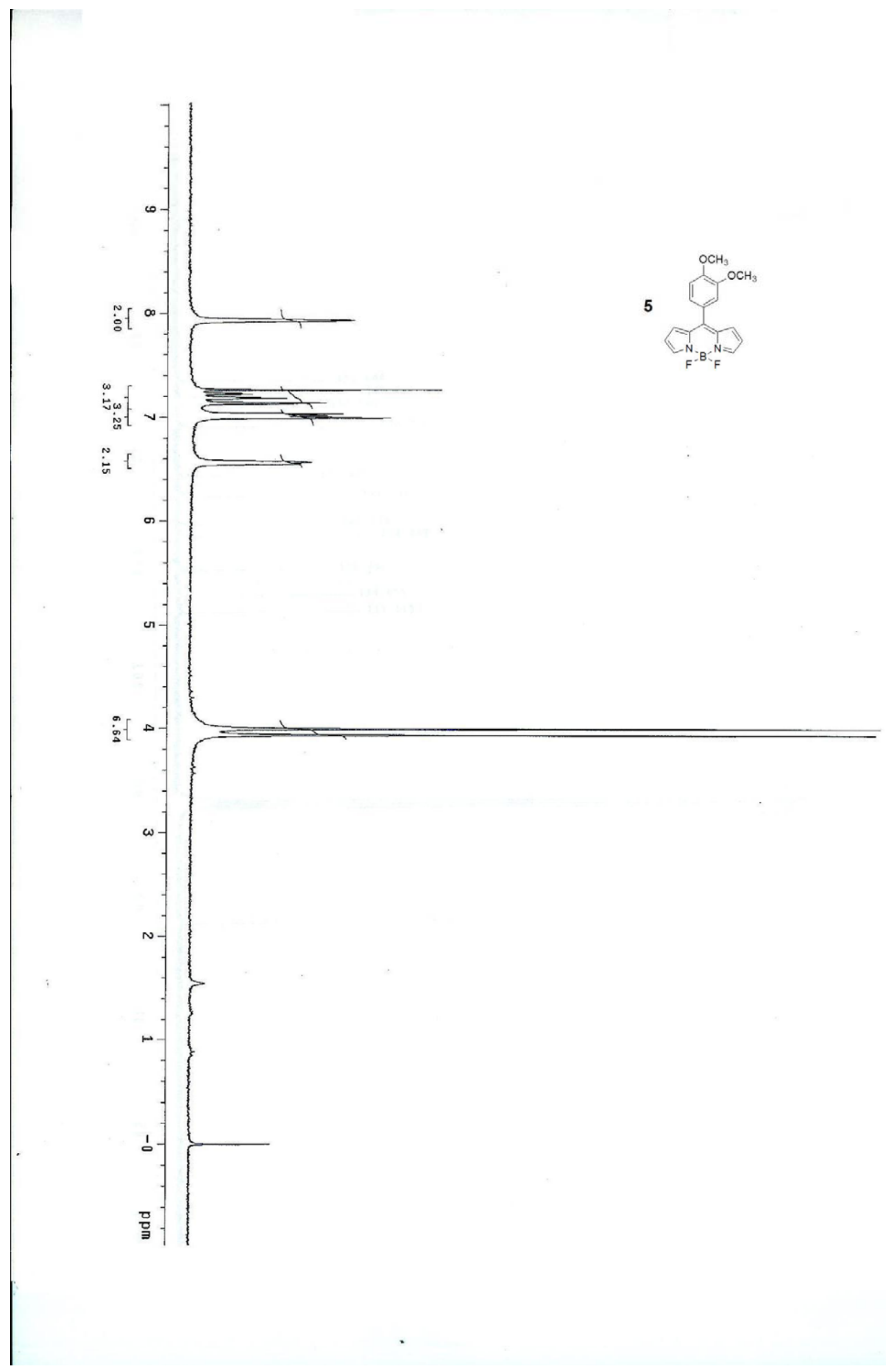


S13

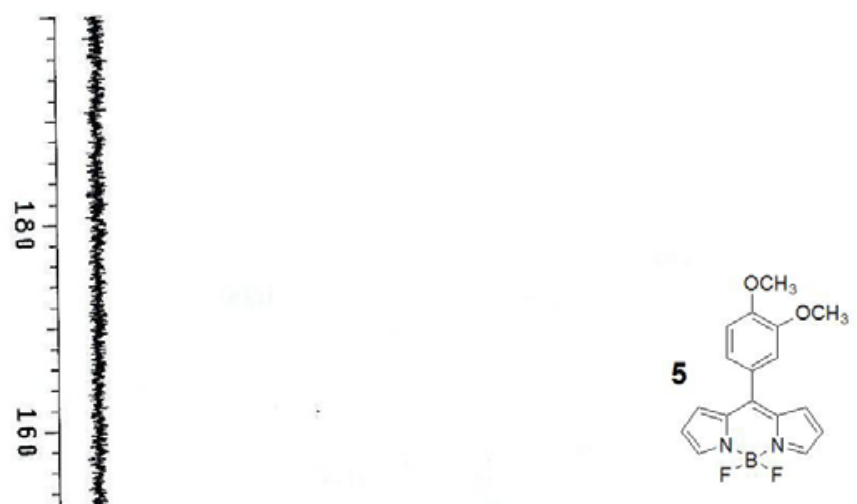

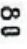

吕-

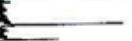

N-1
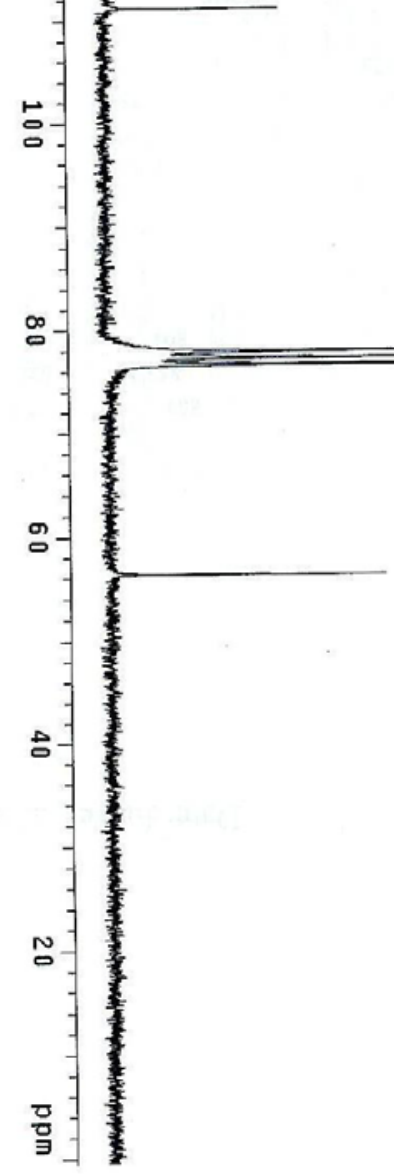


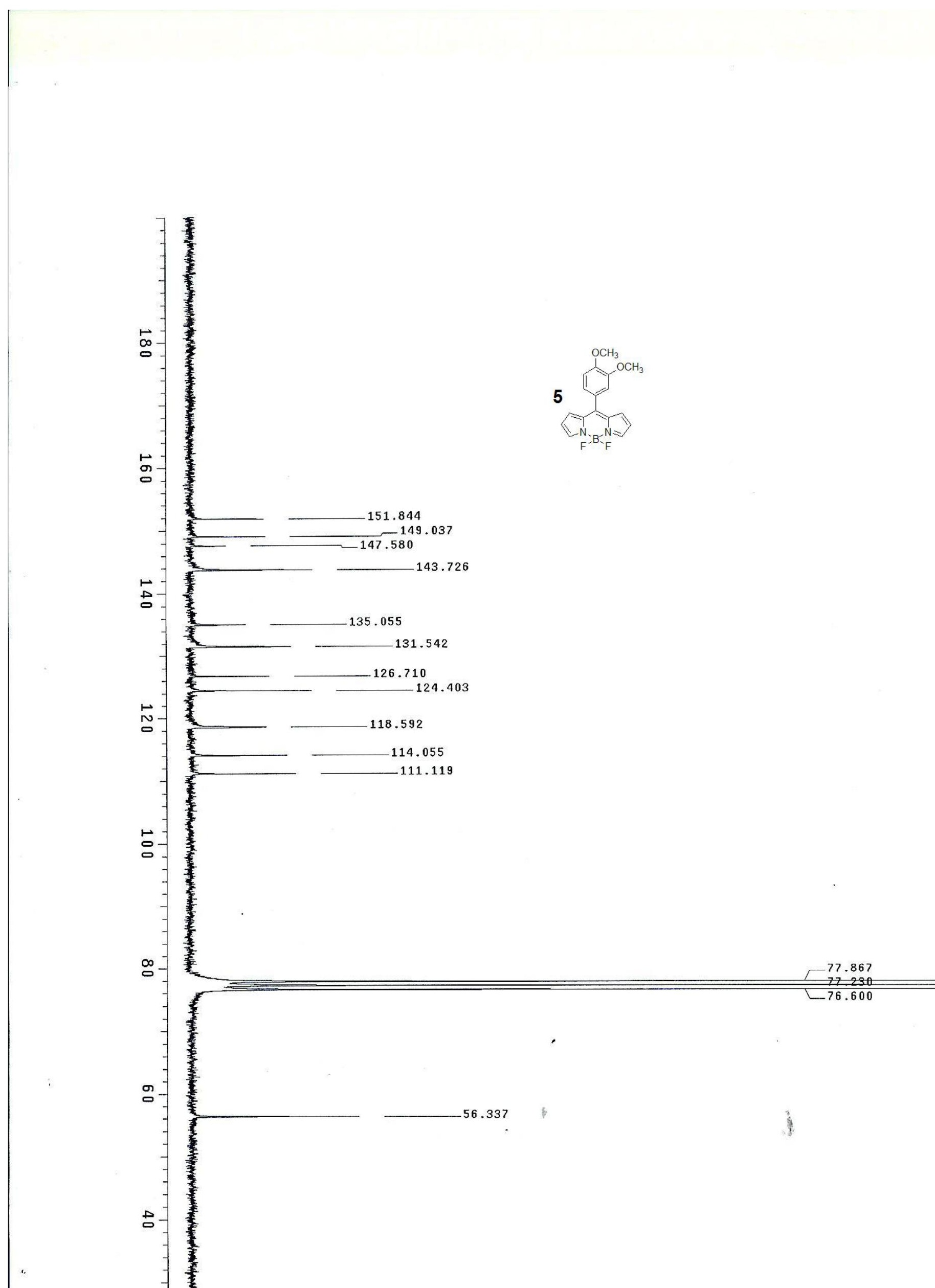




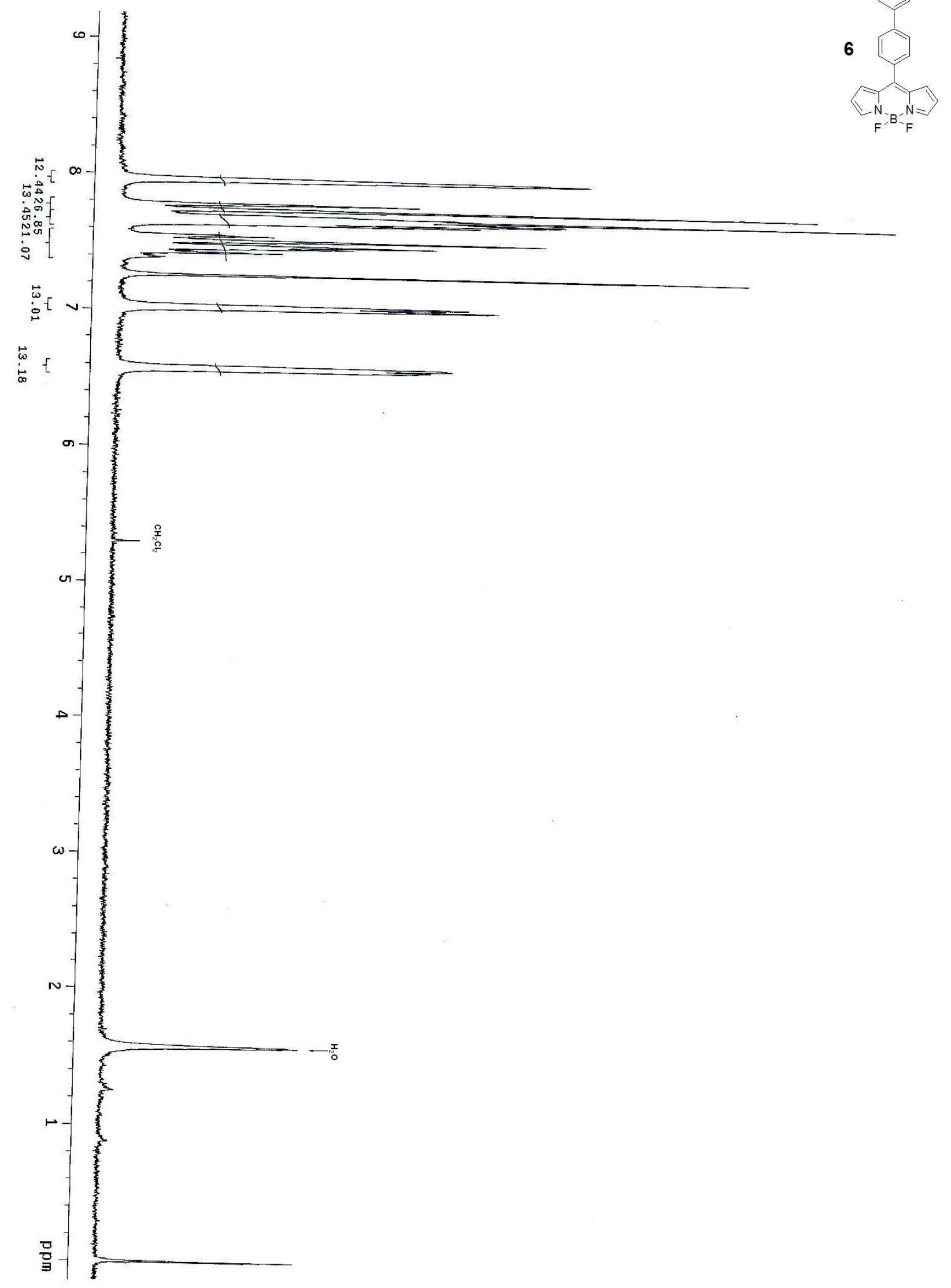


S16

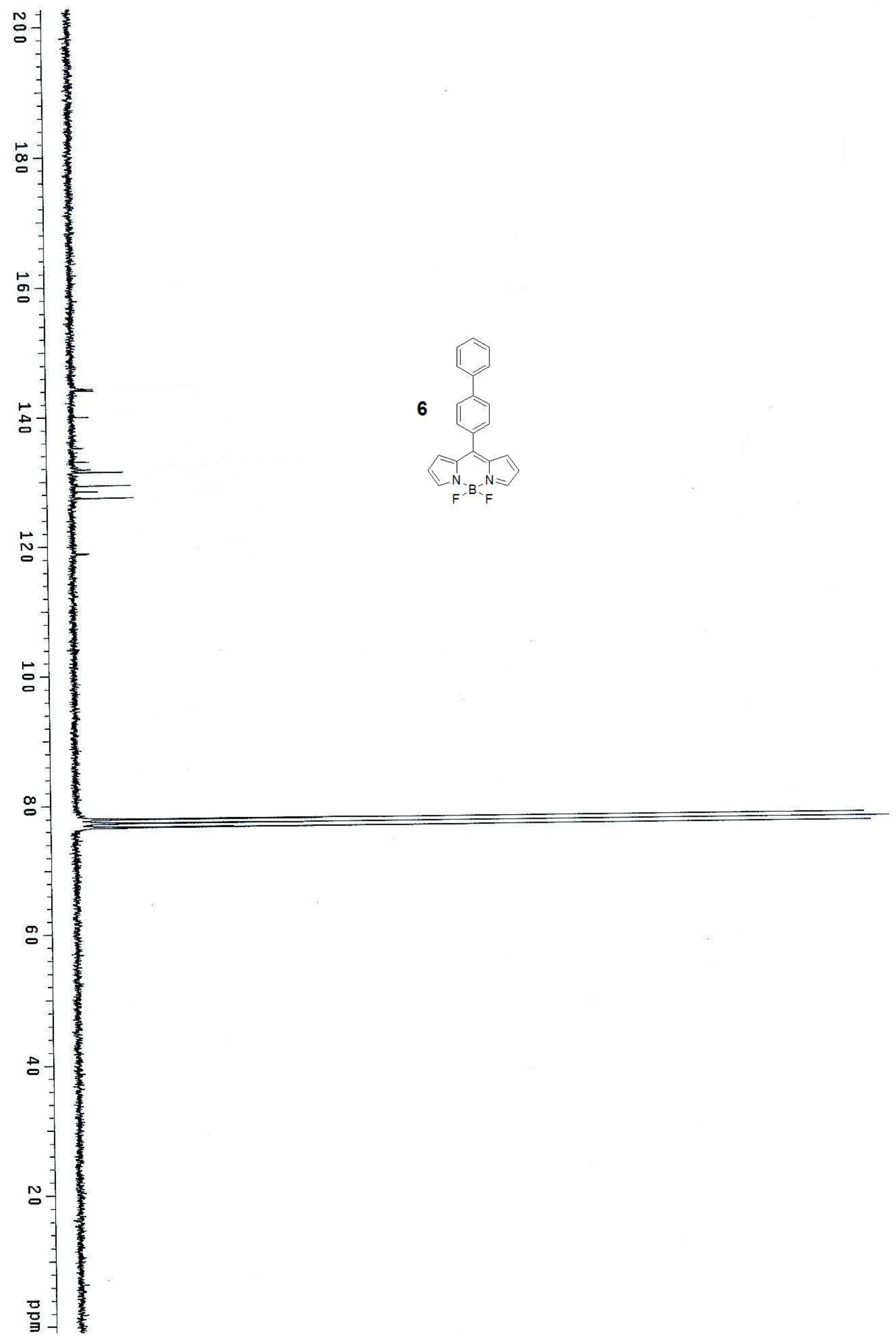




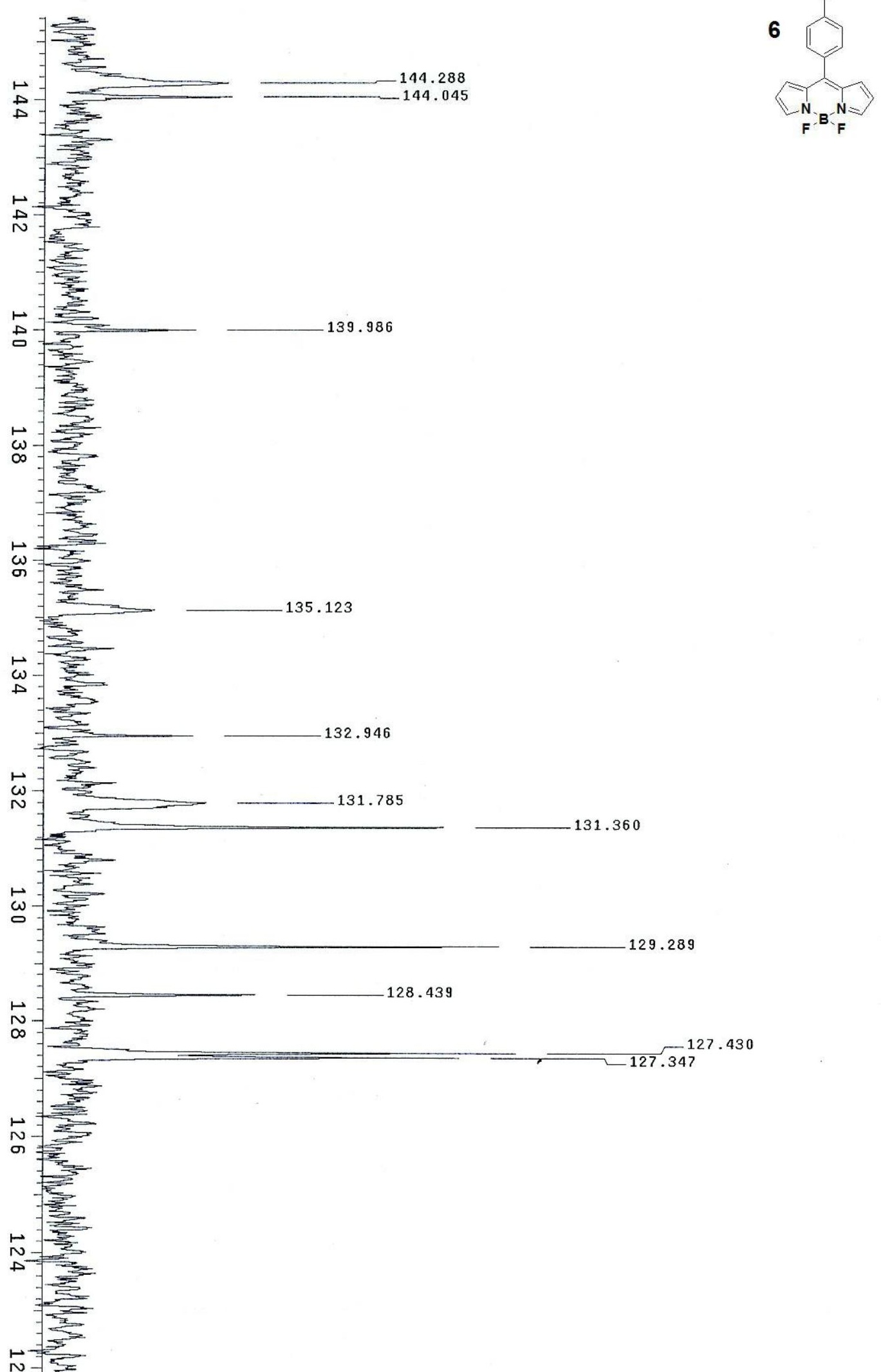




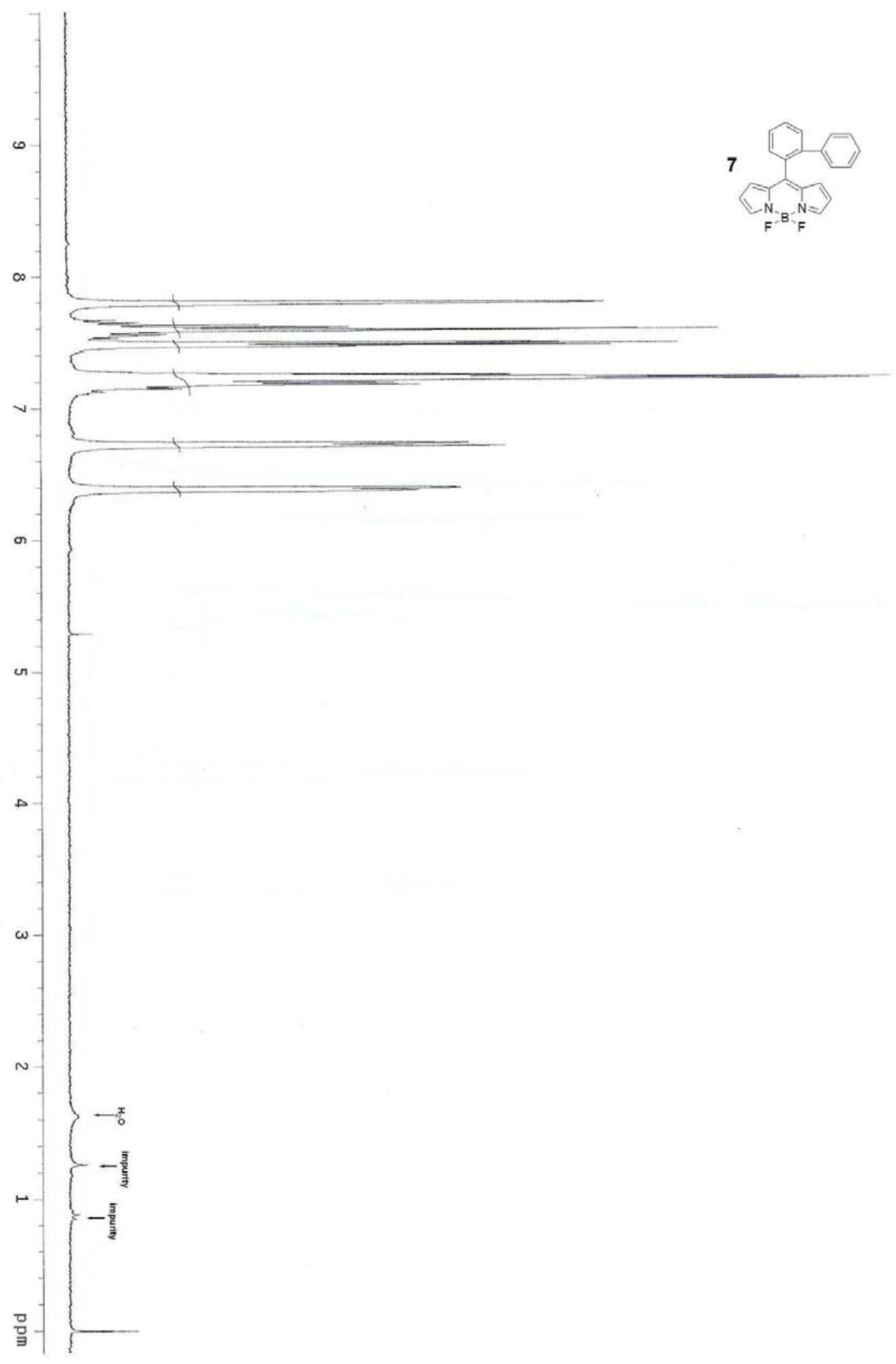


S19

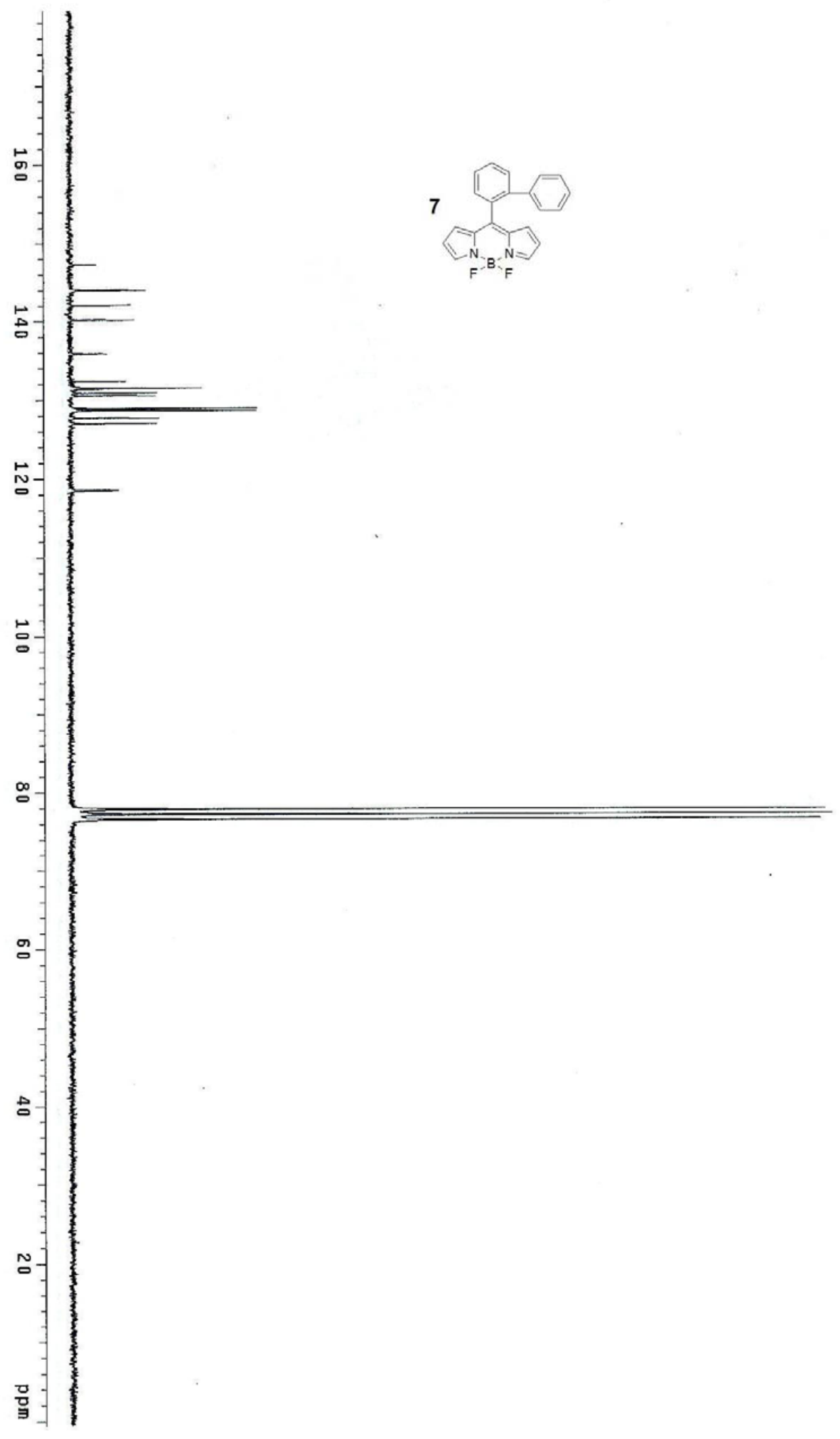



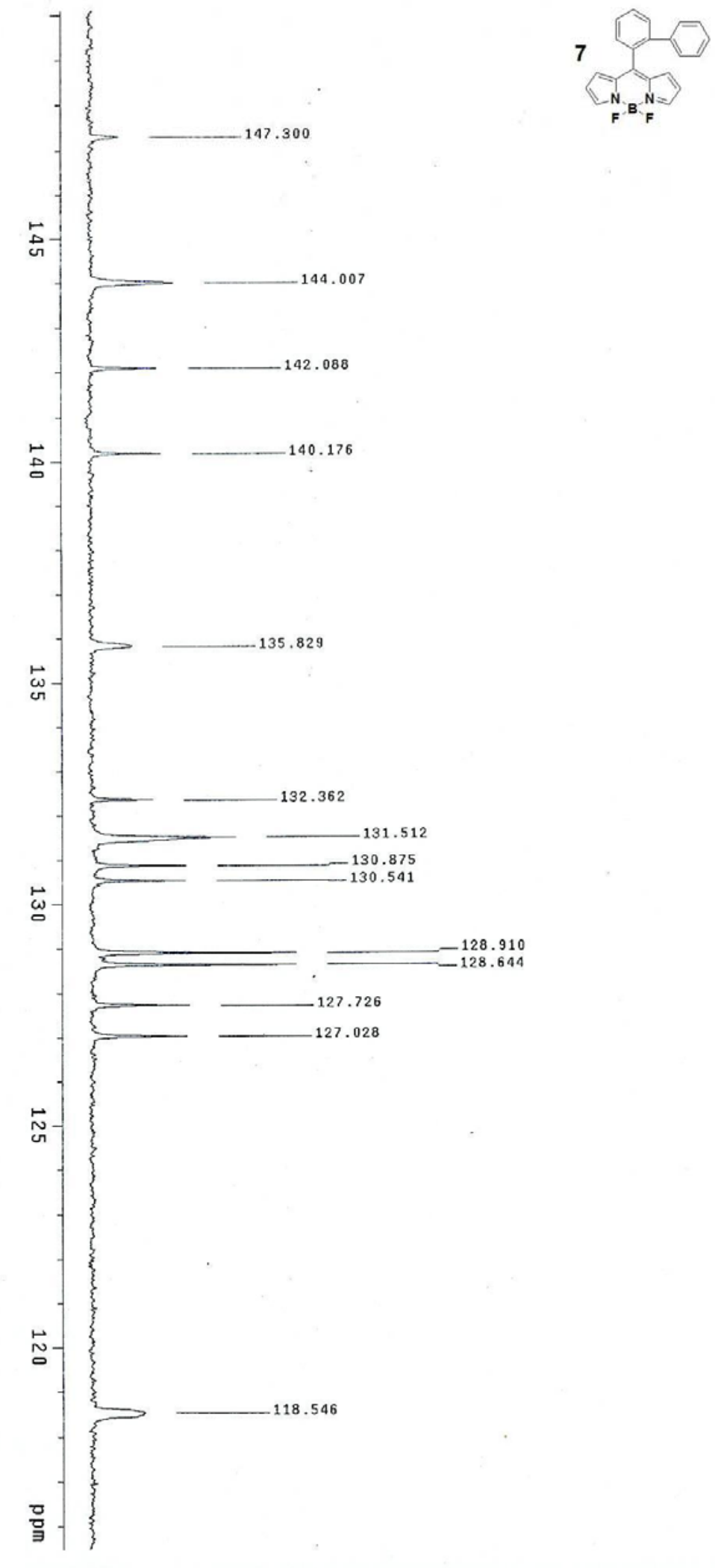


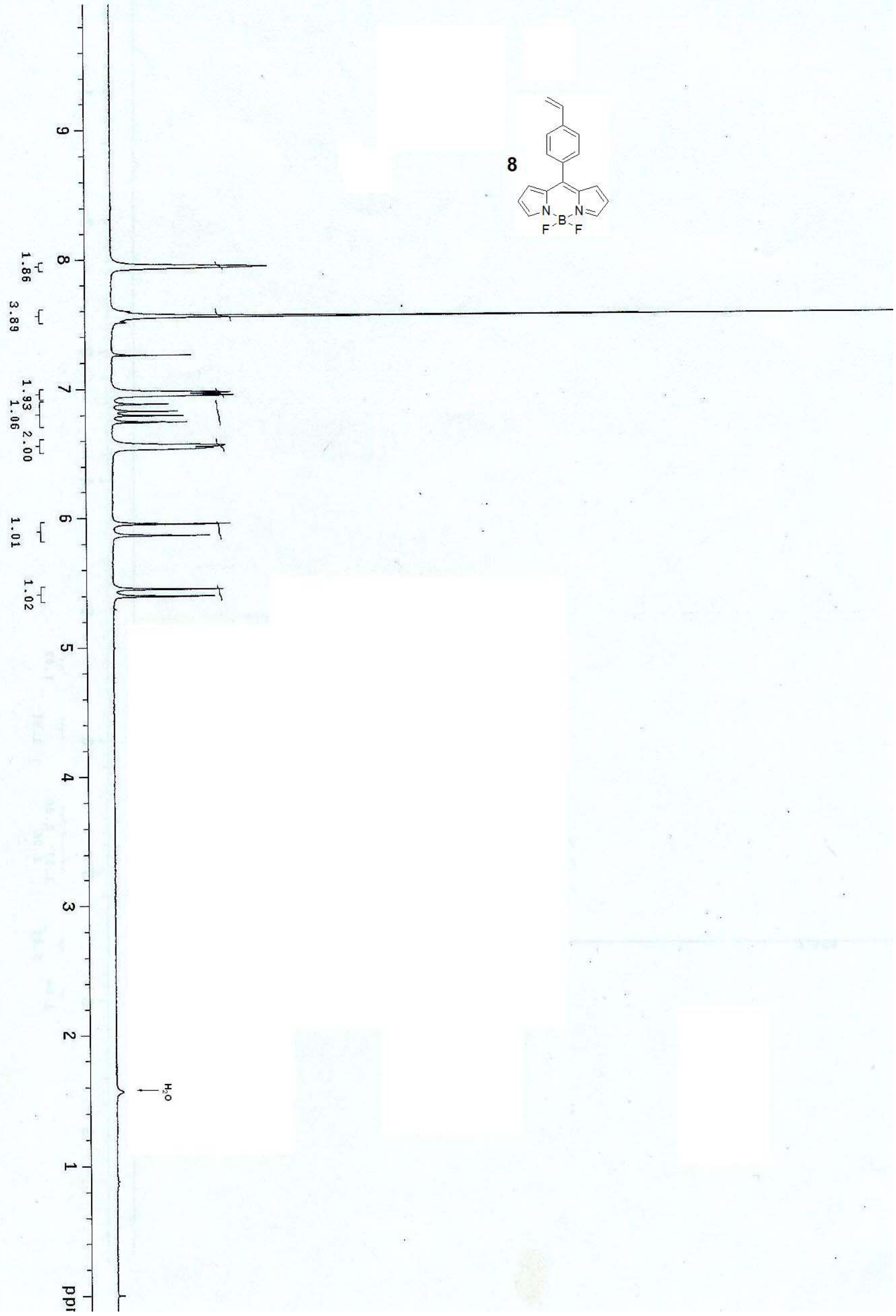




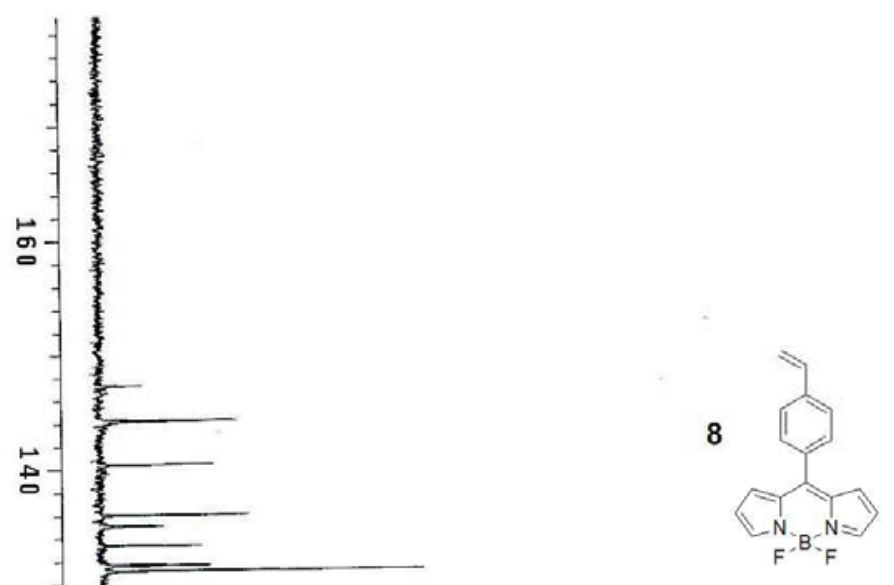




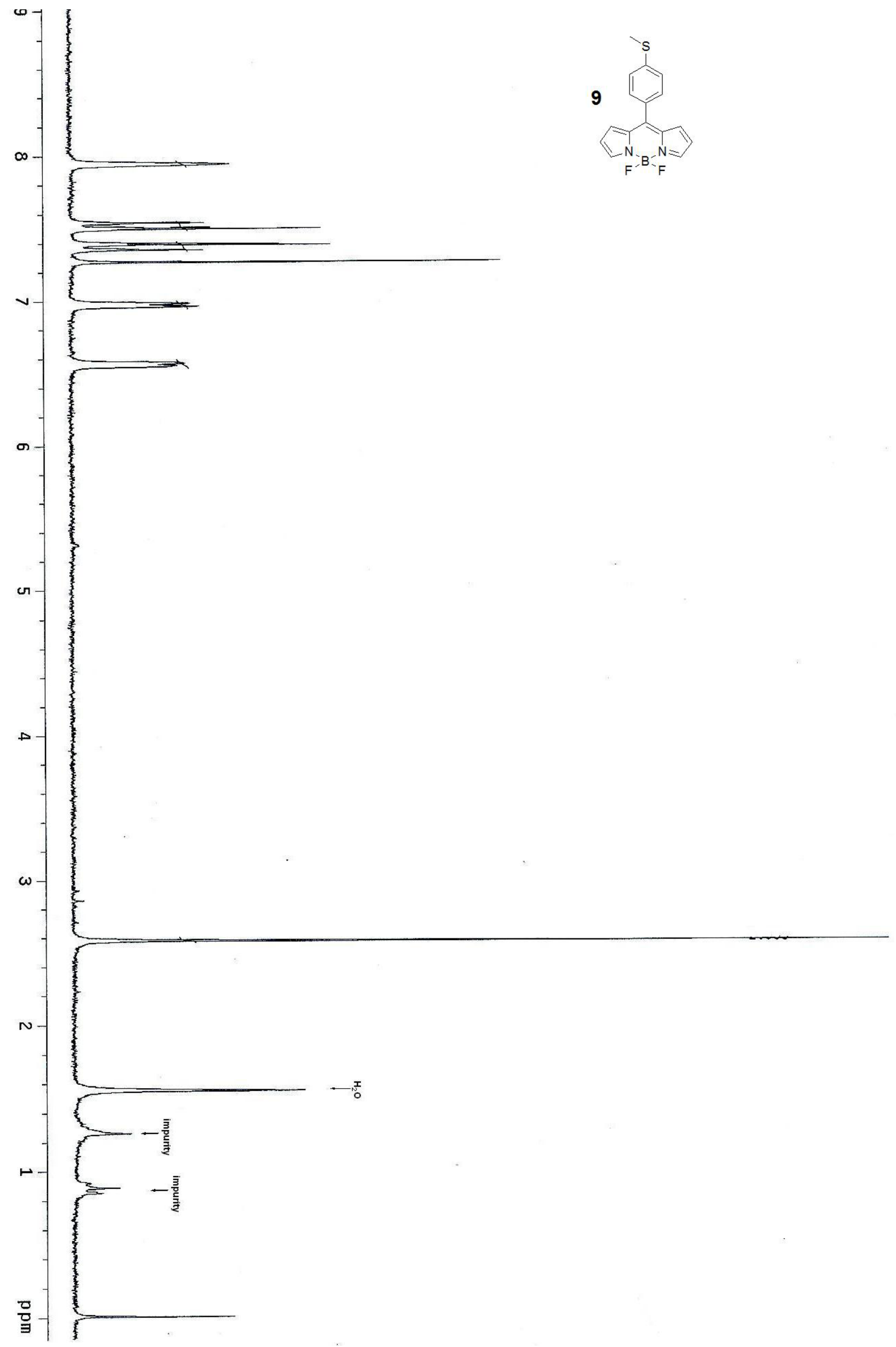




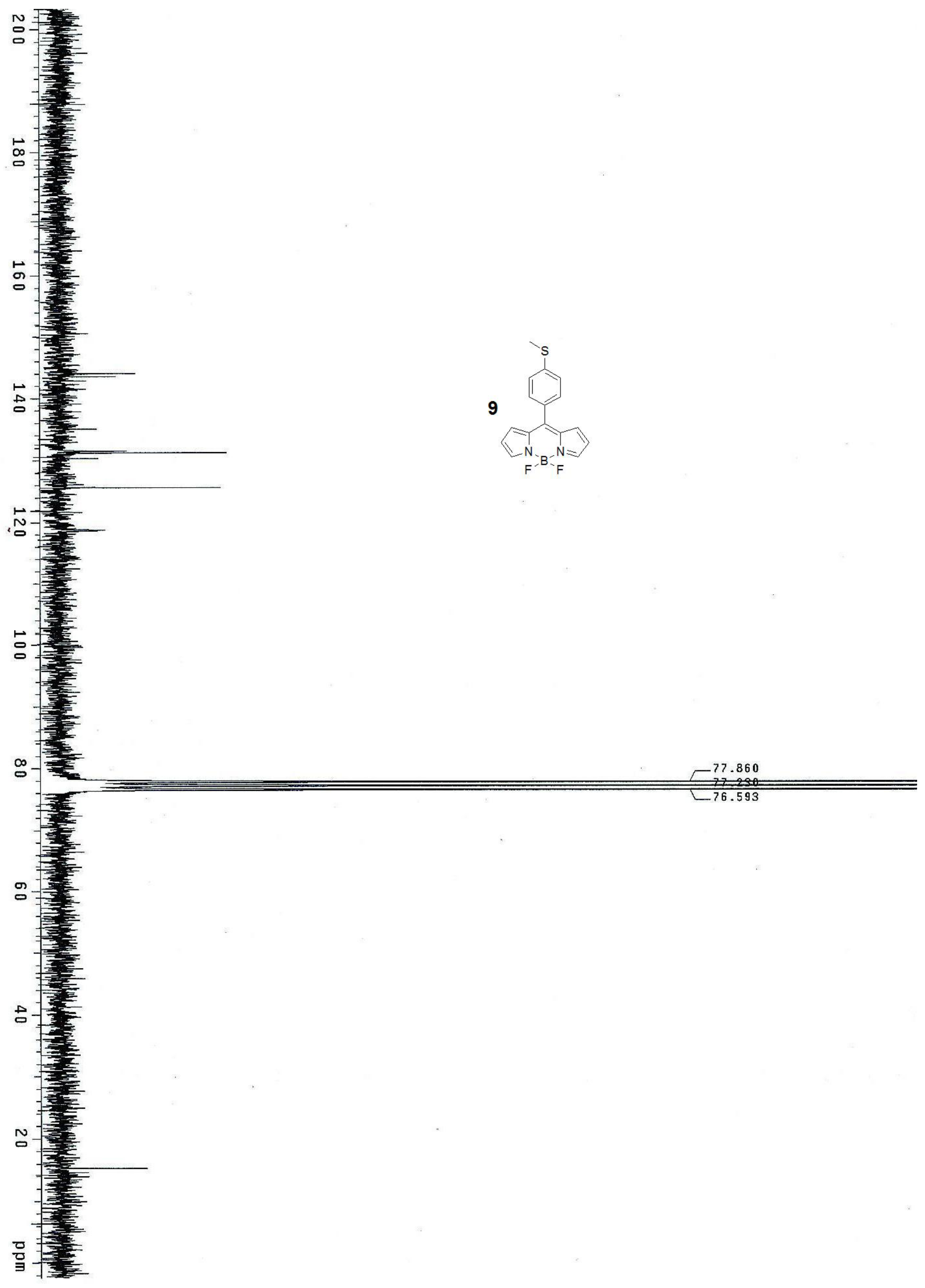




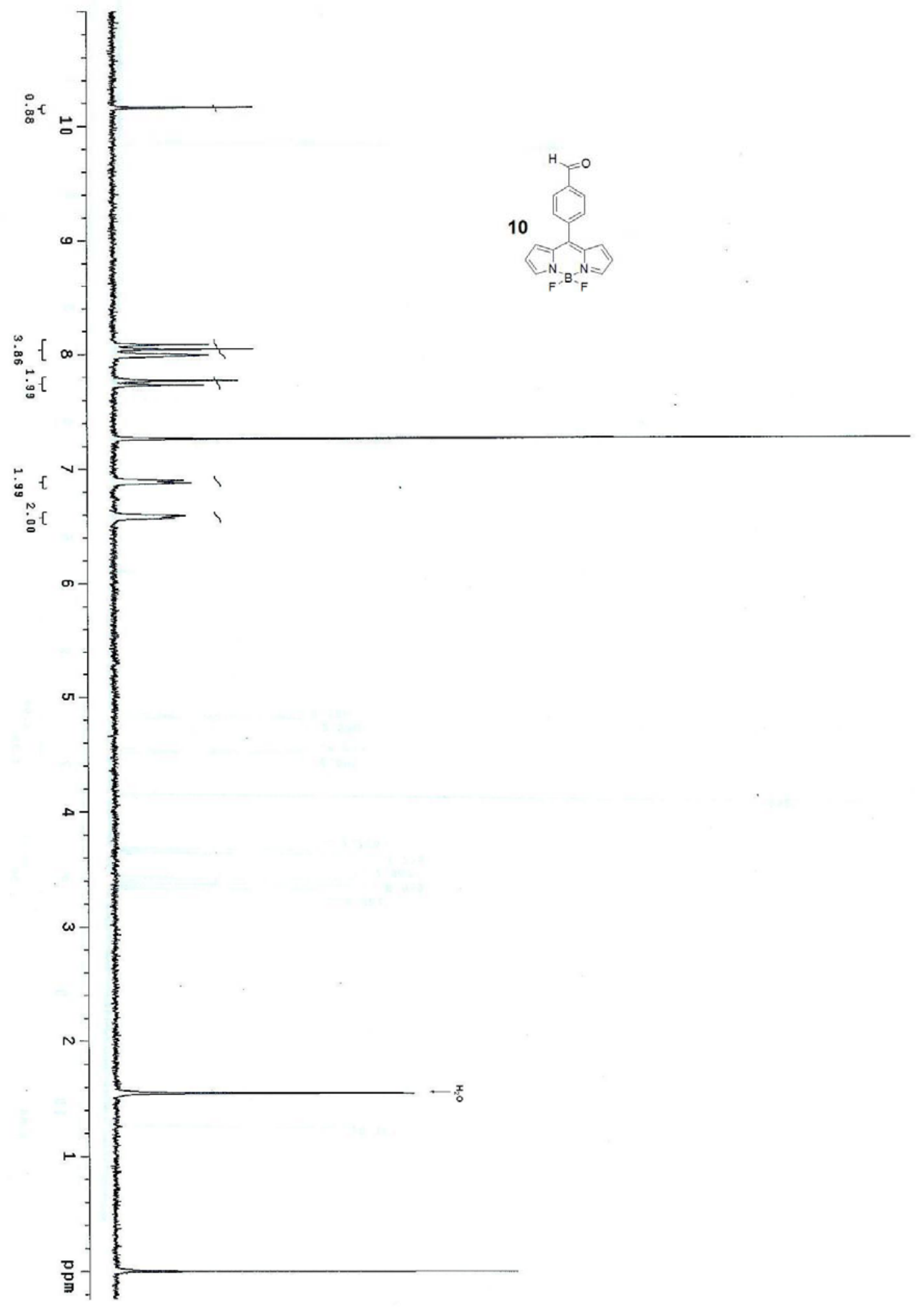




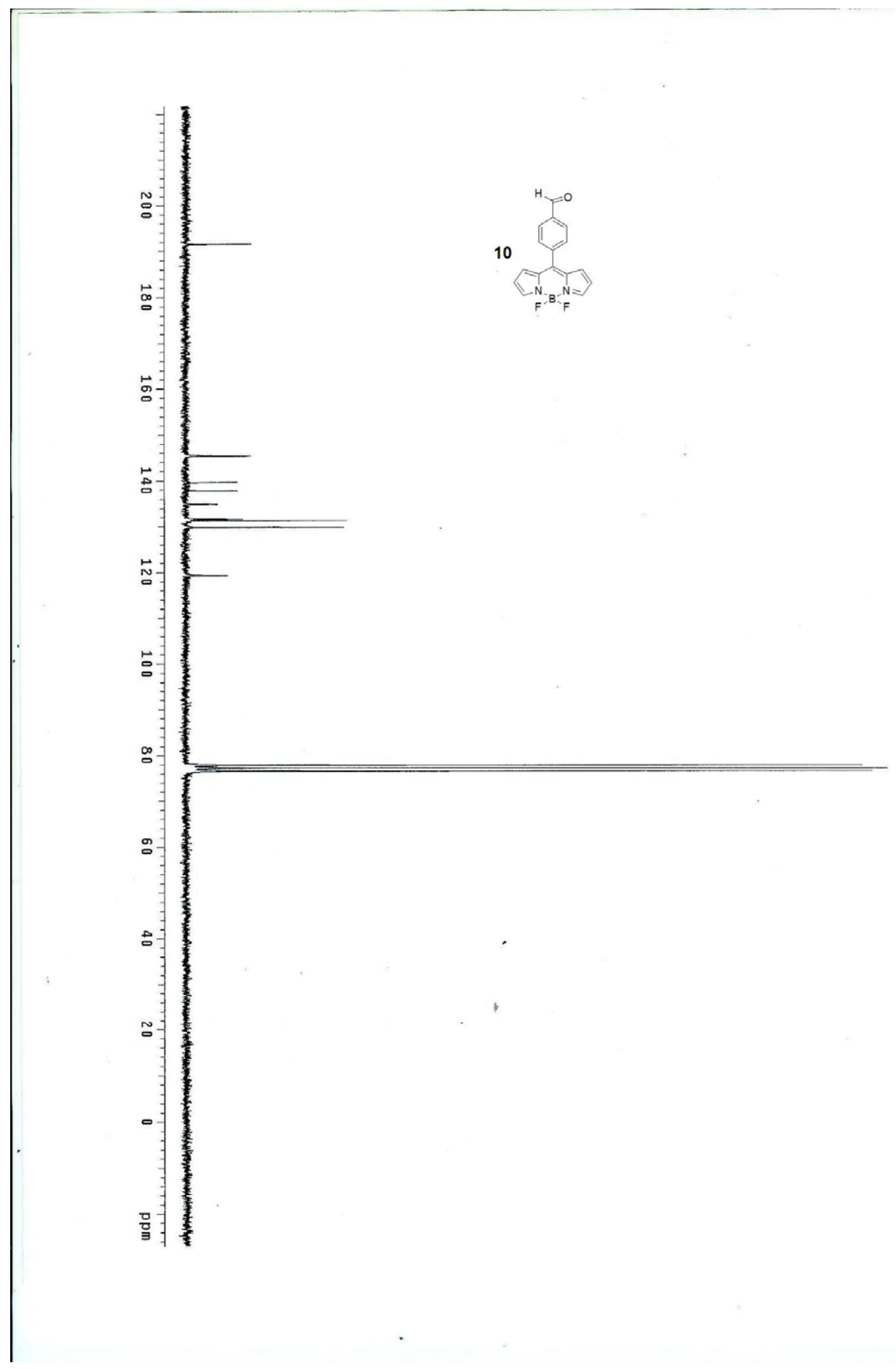




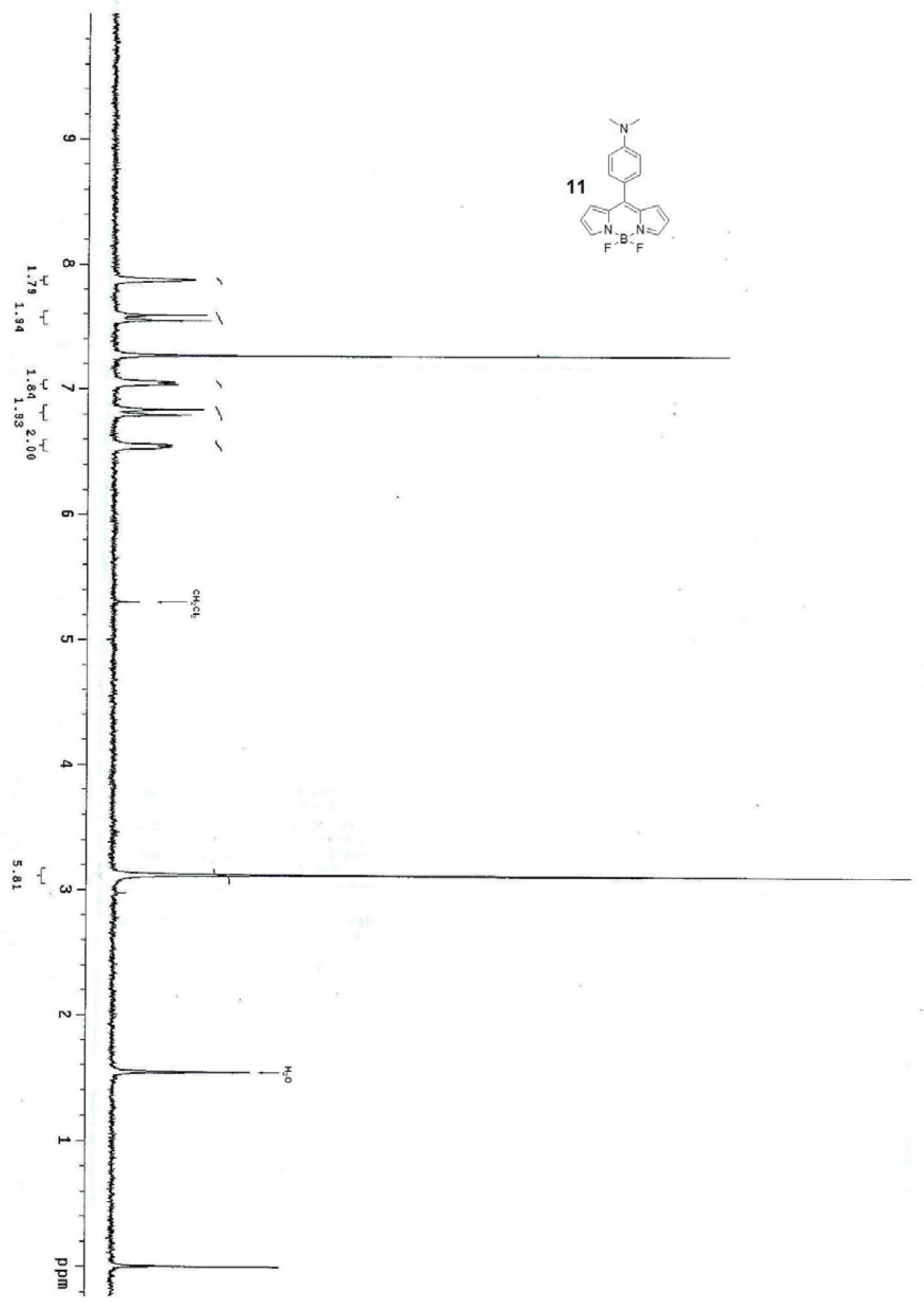




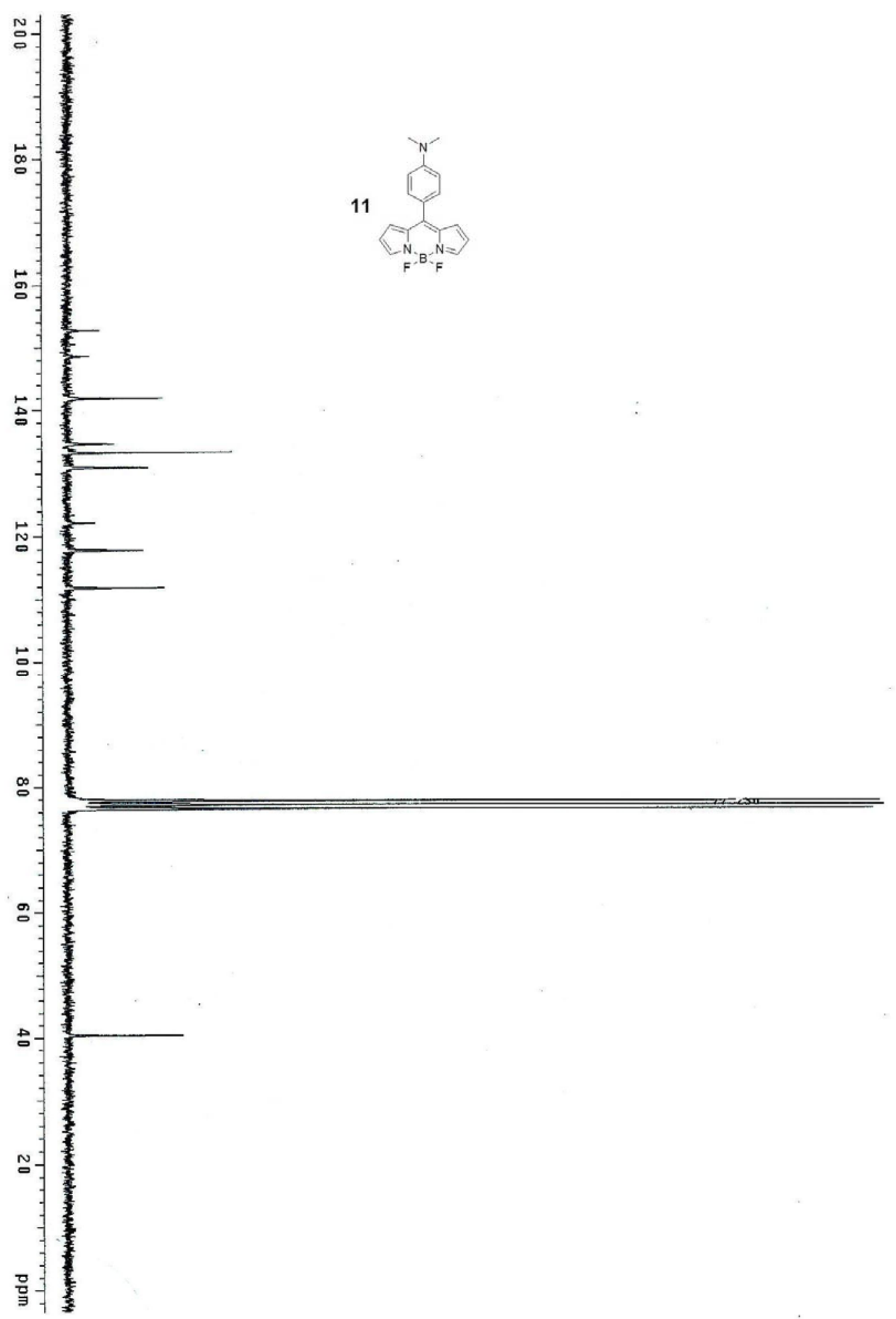


S29

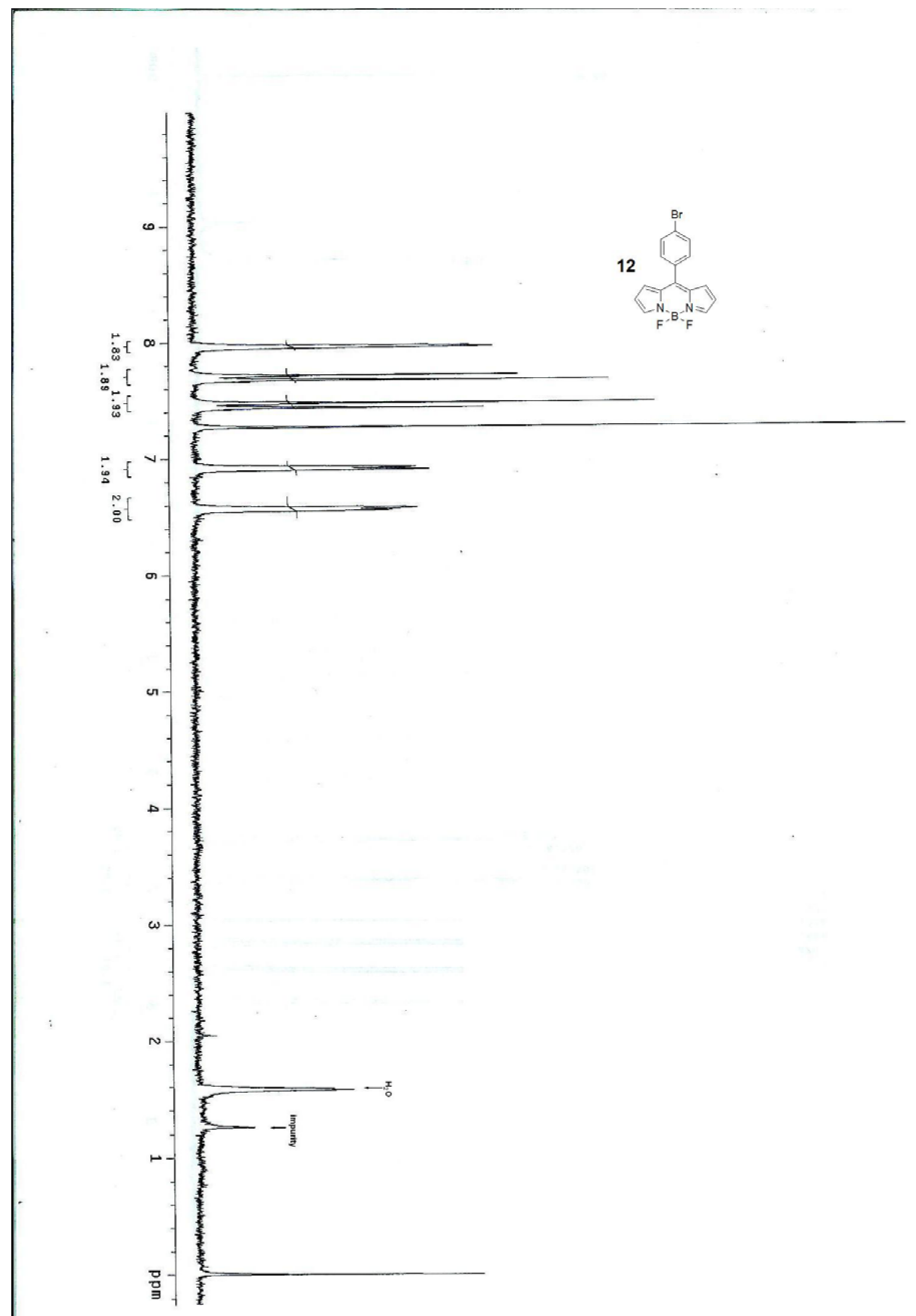




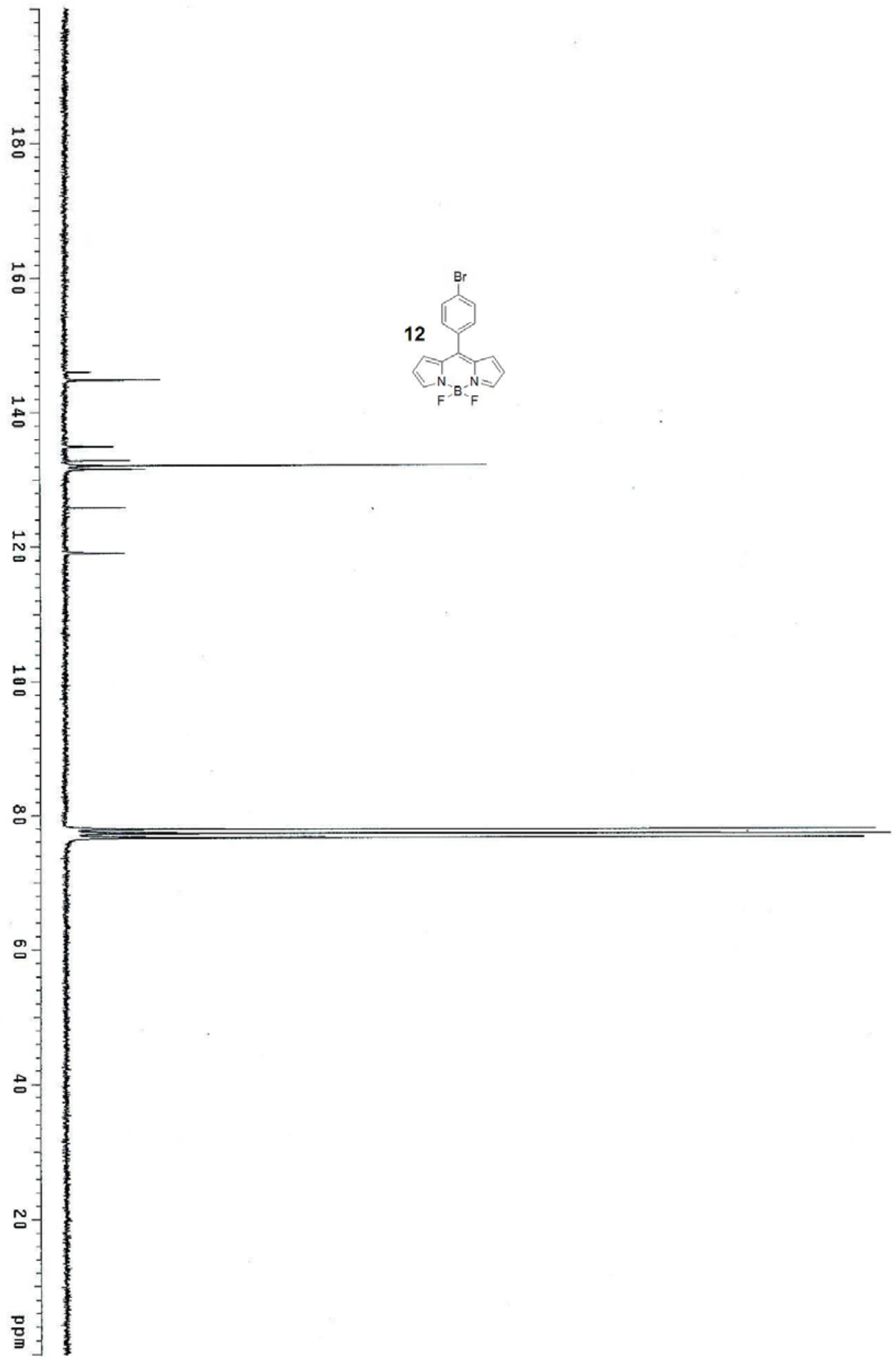



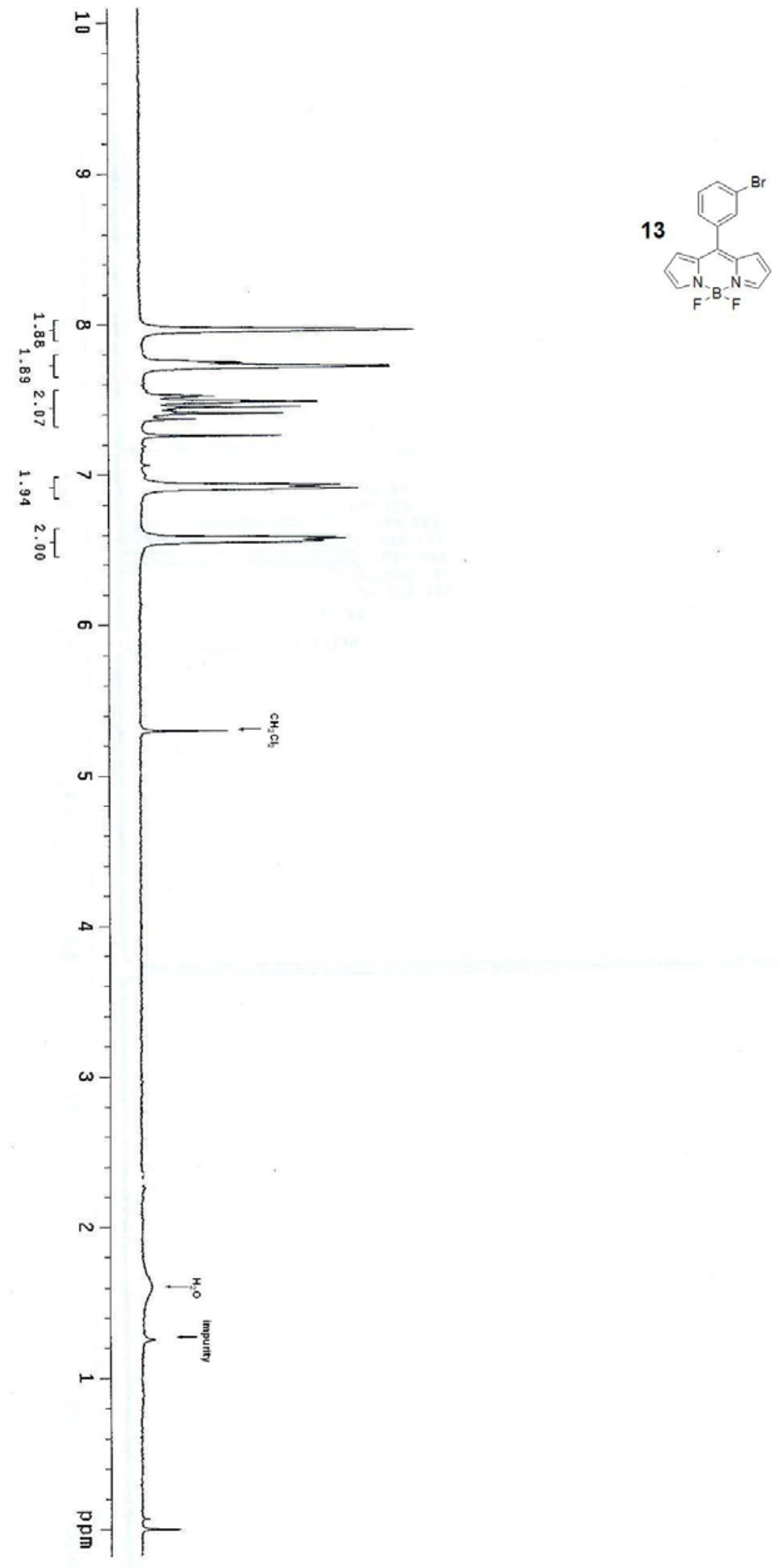


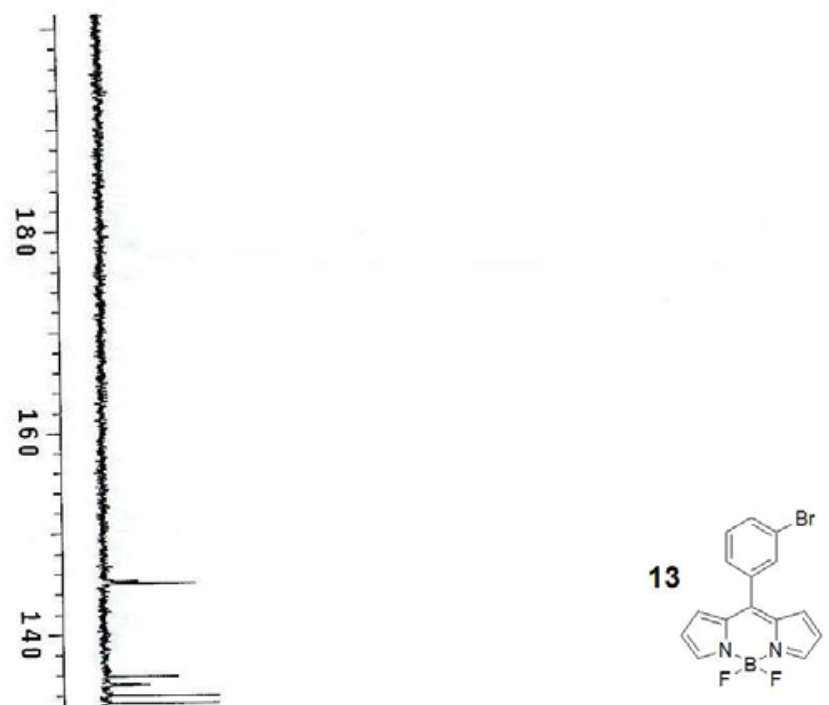




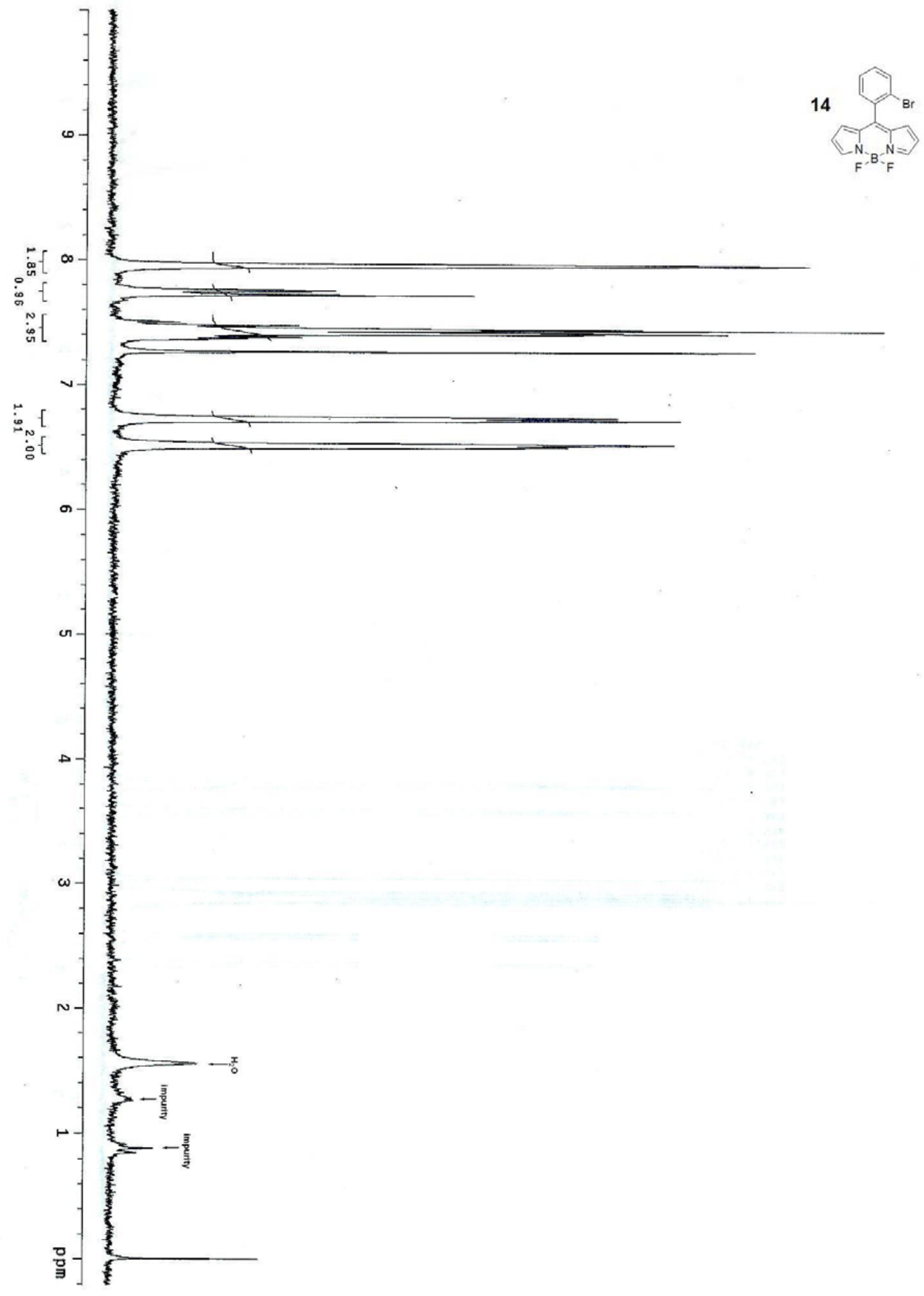




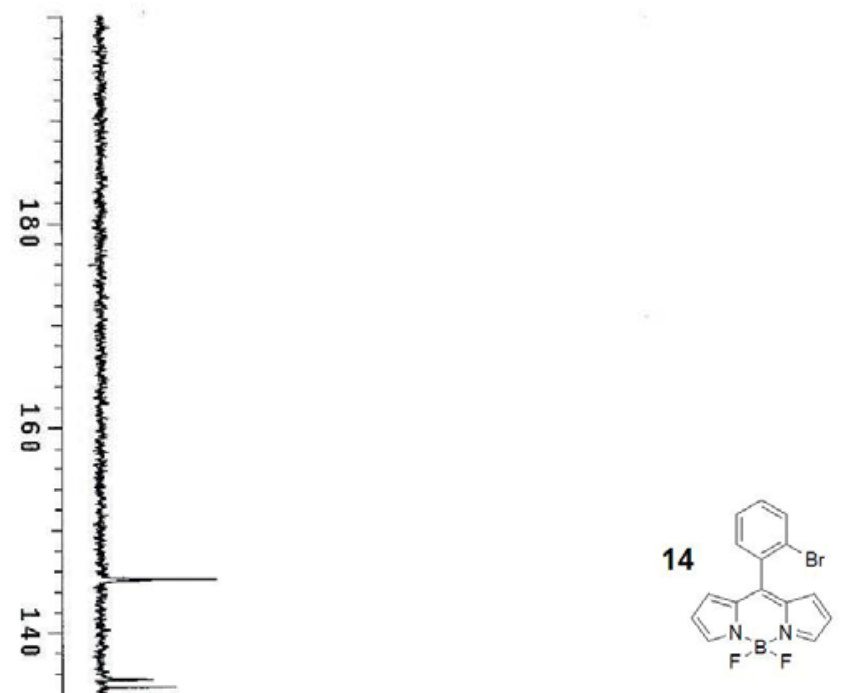

-

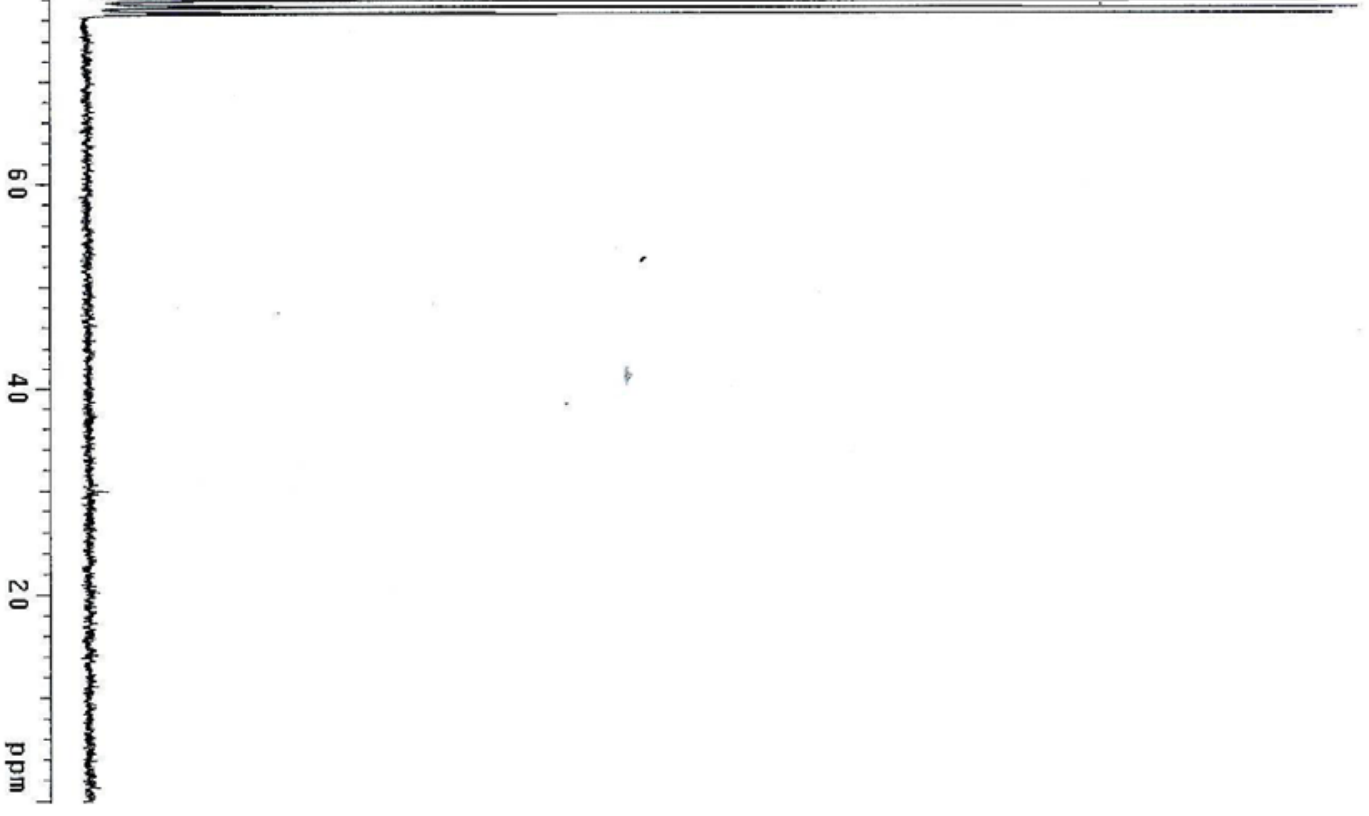


S35

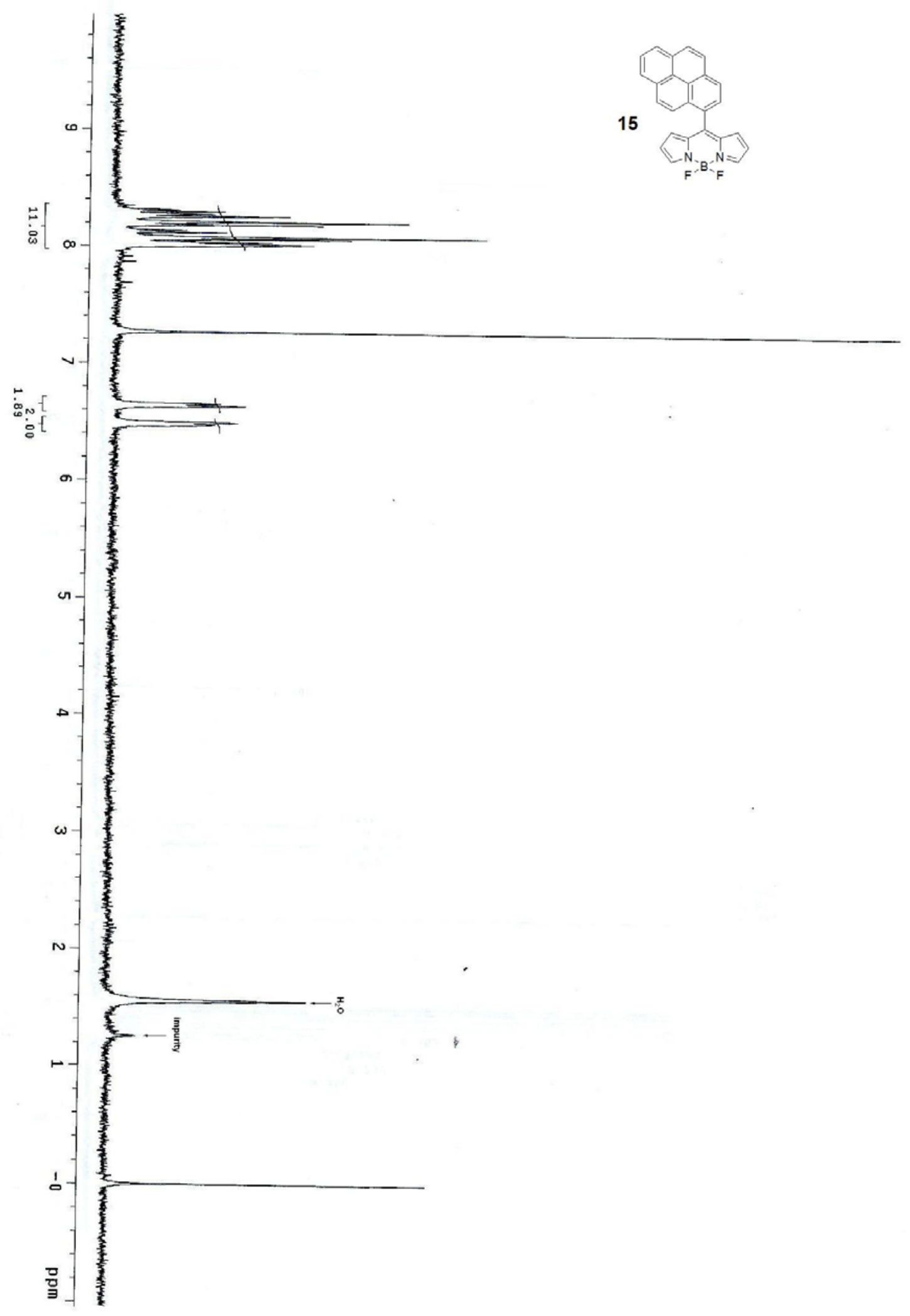




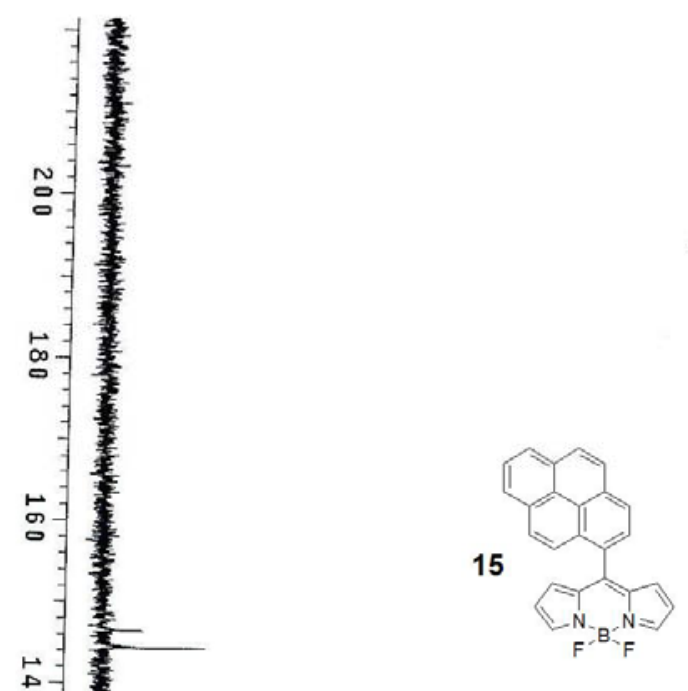

I-

ㅇ․

我-

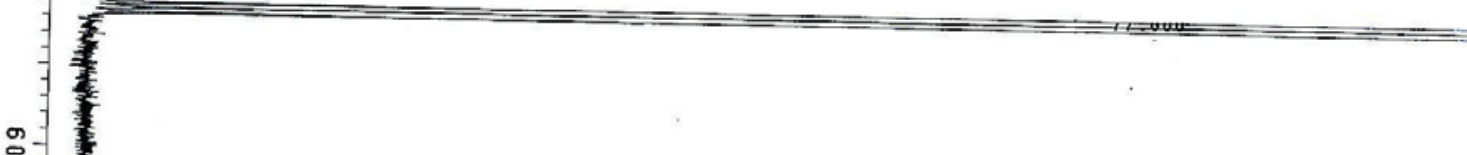



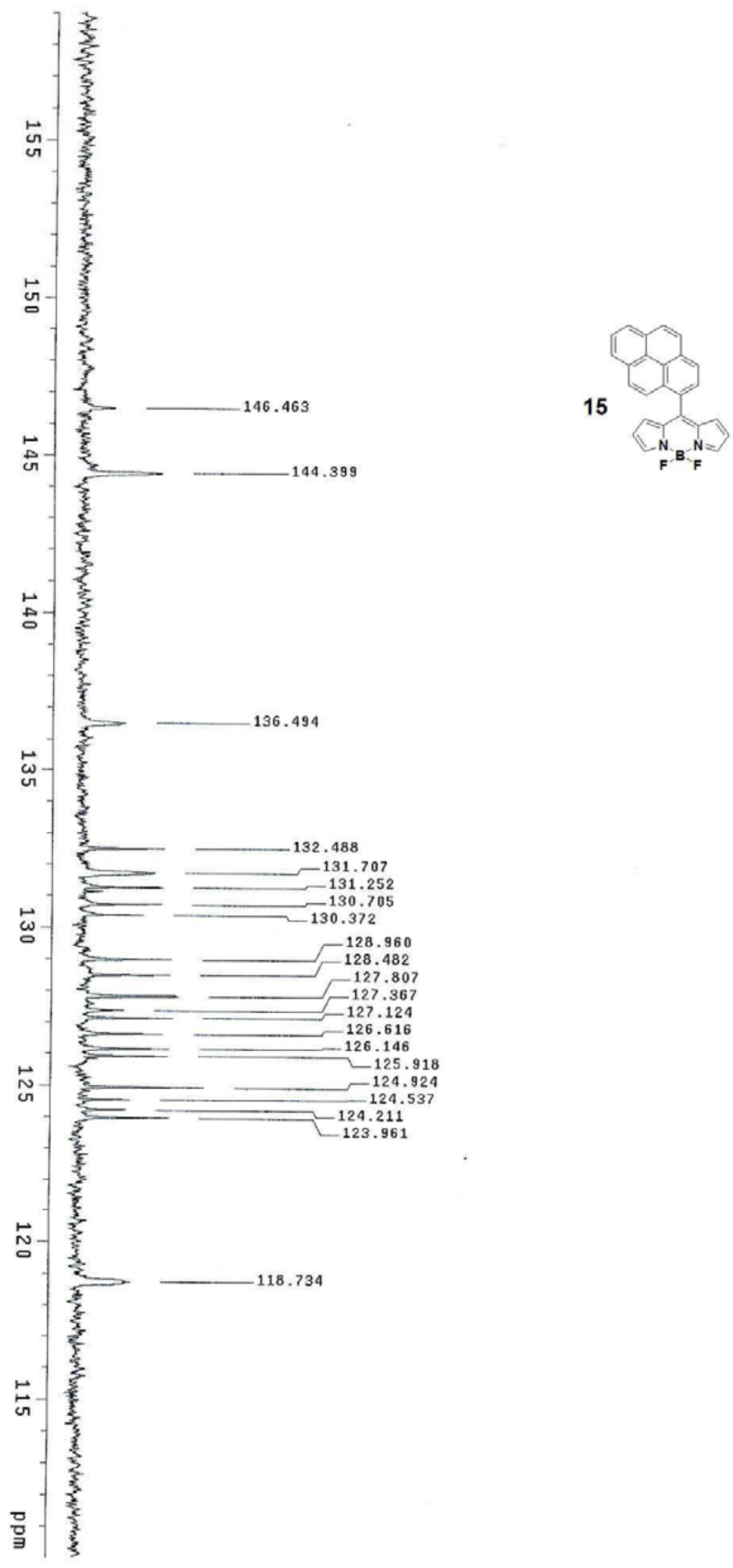


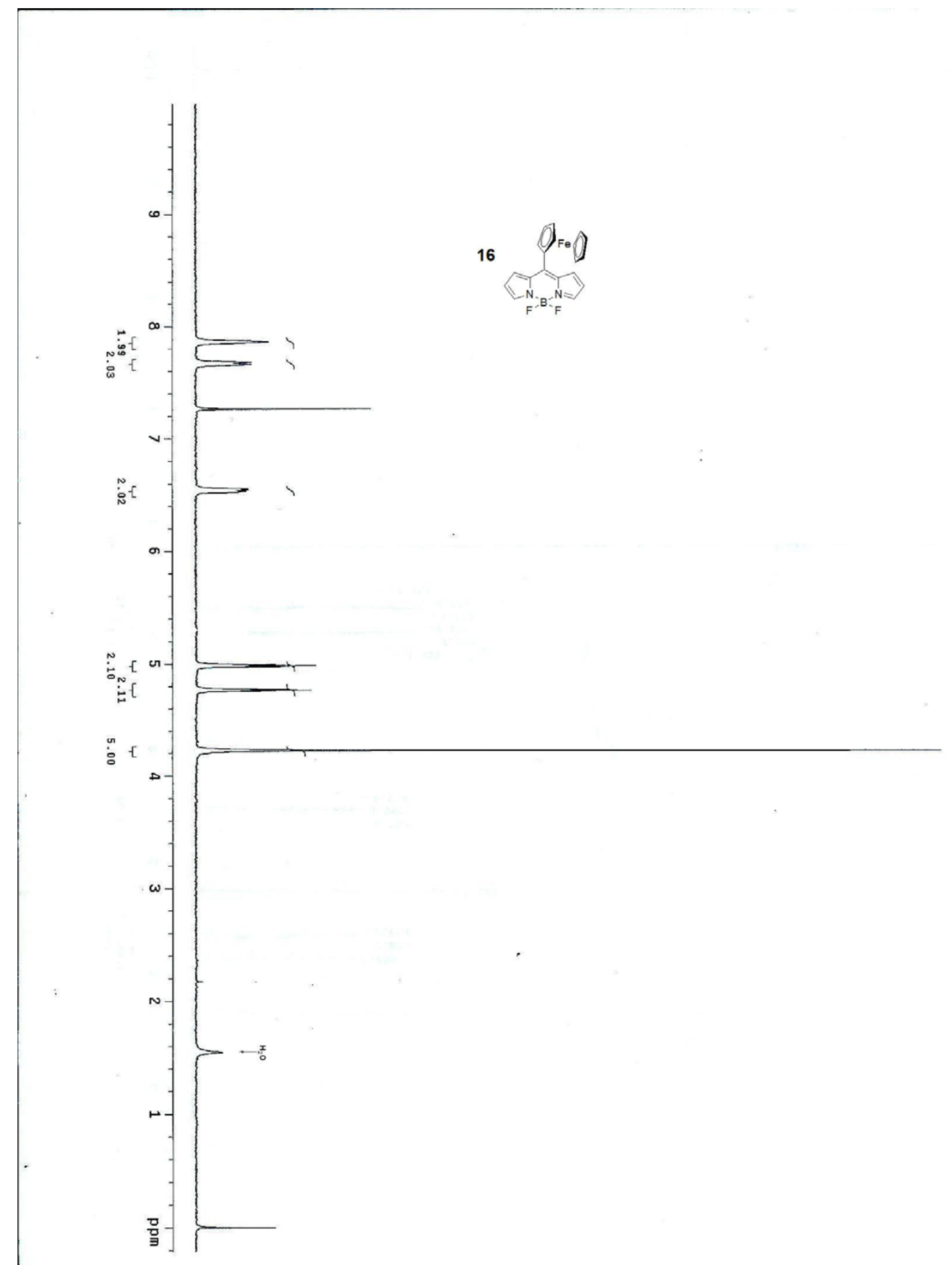




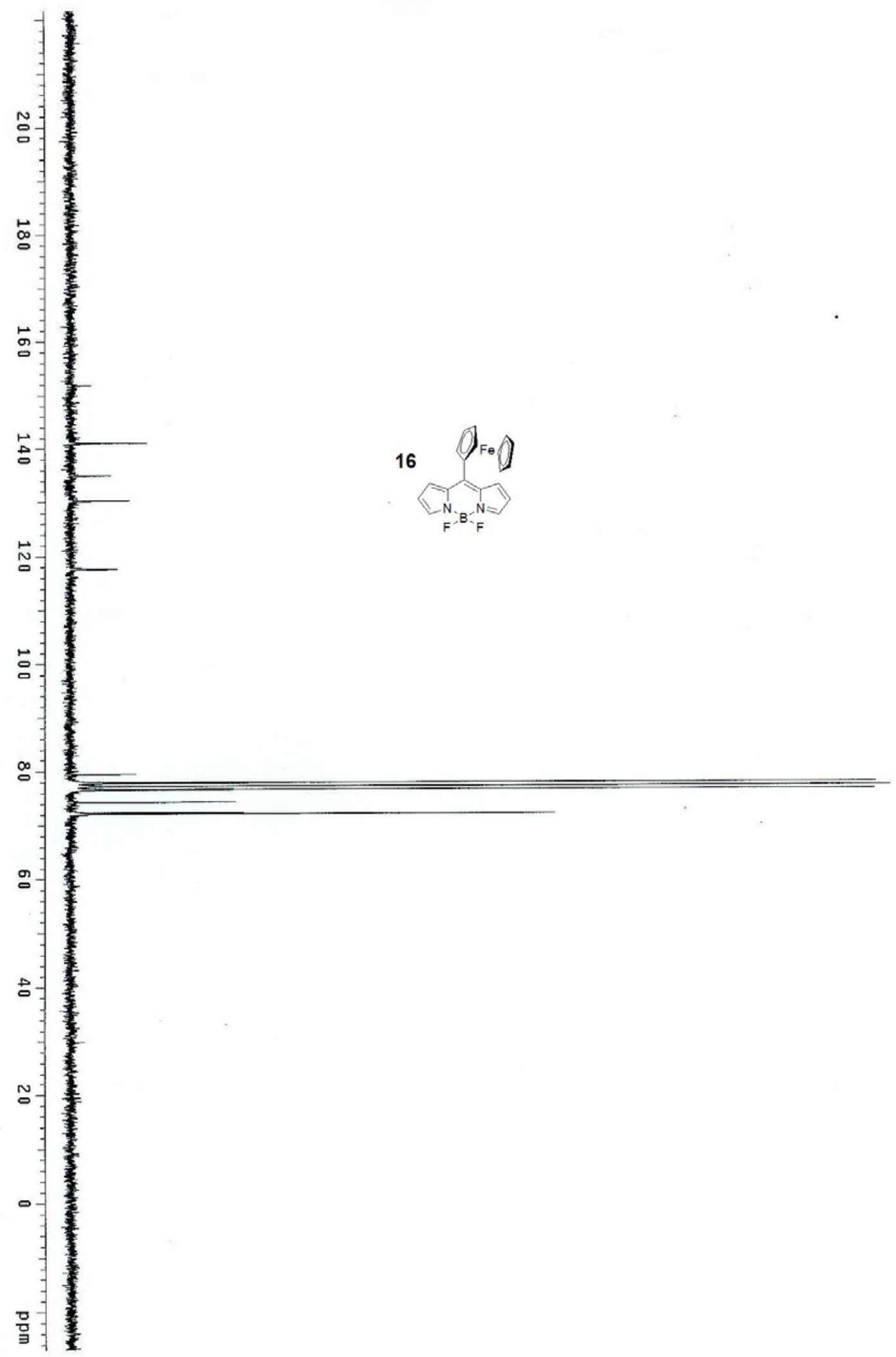


S40

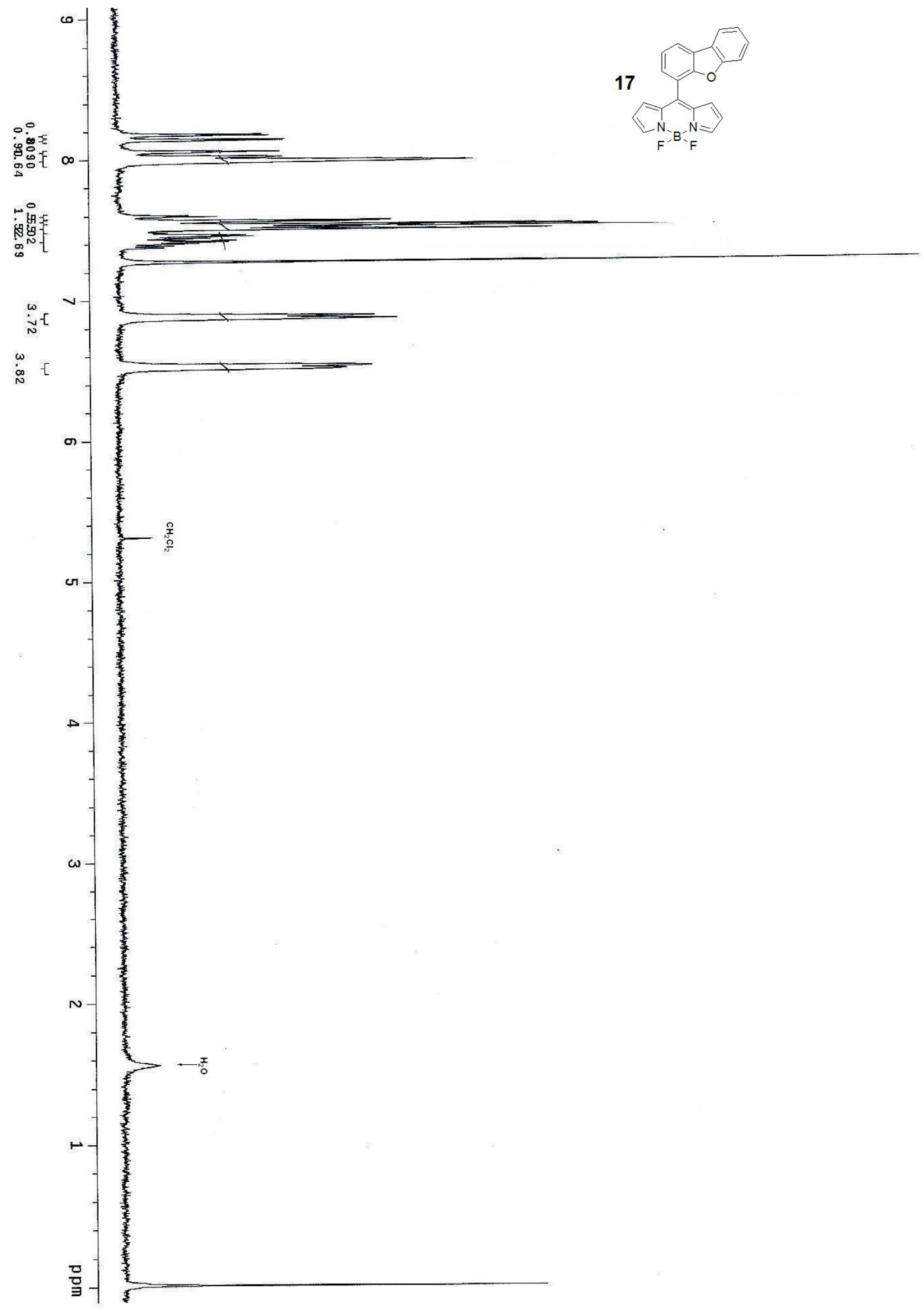




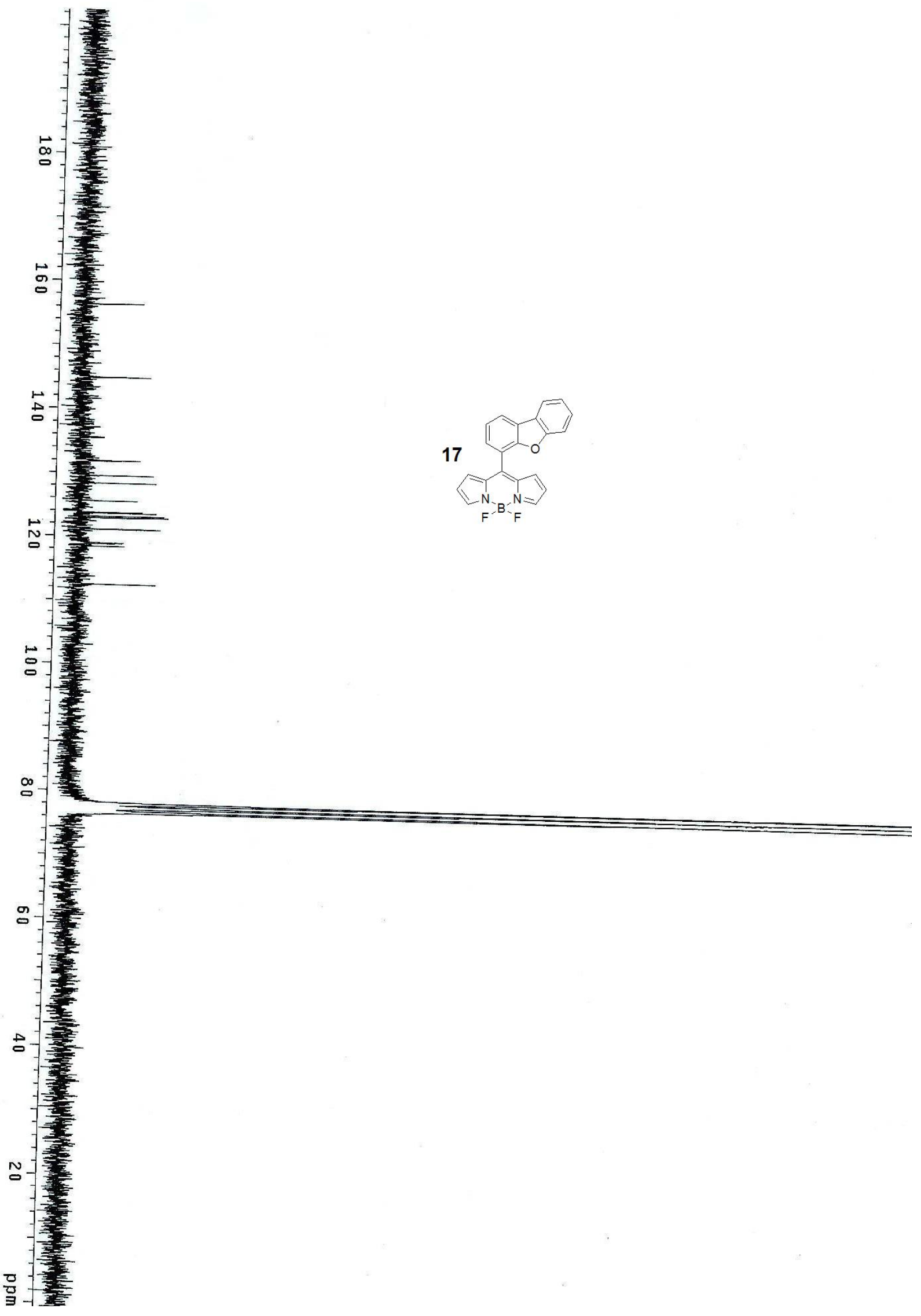



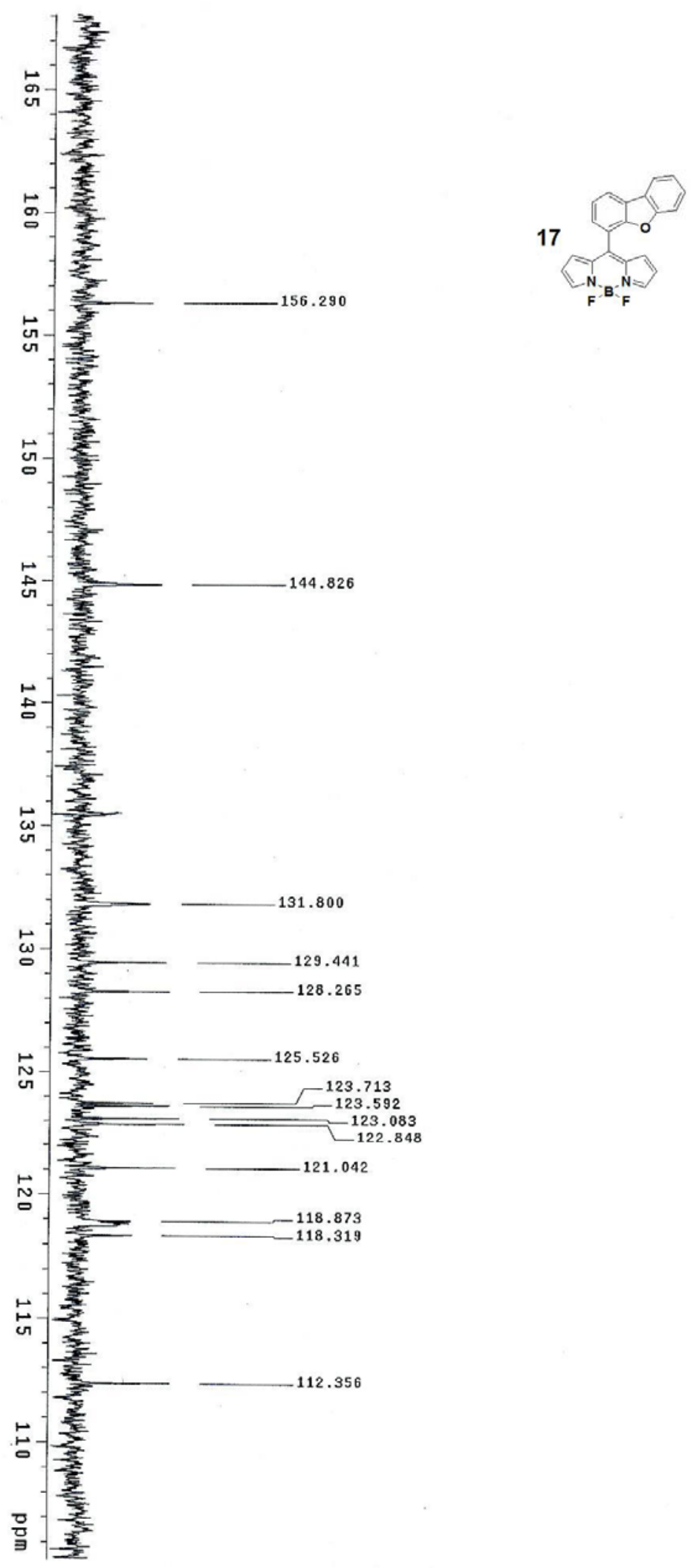


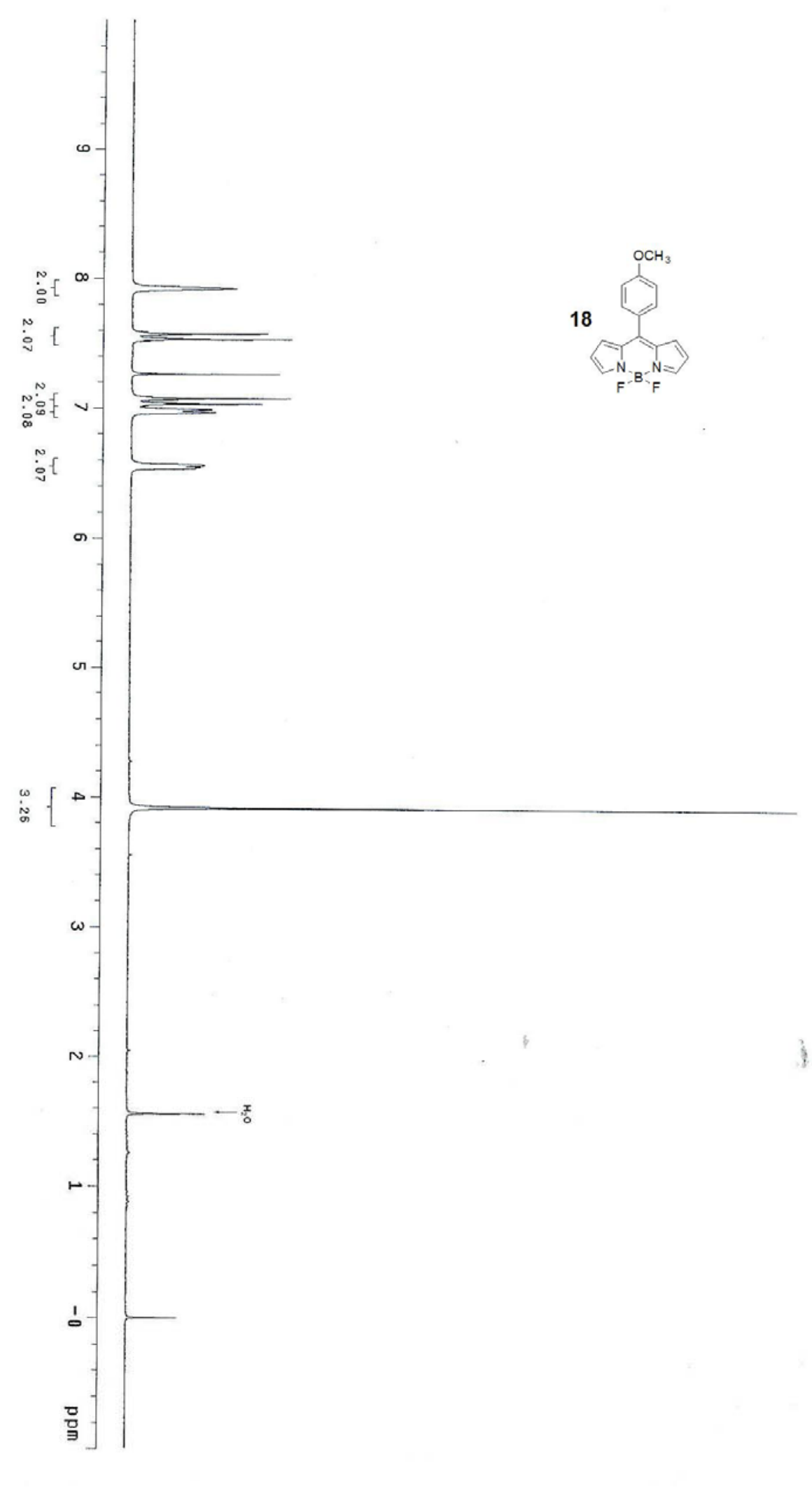




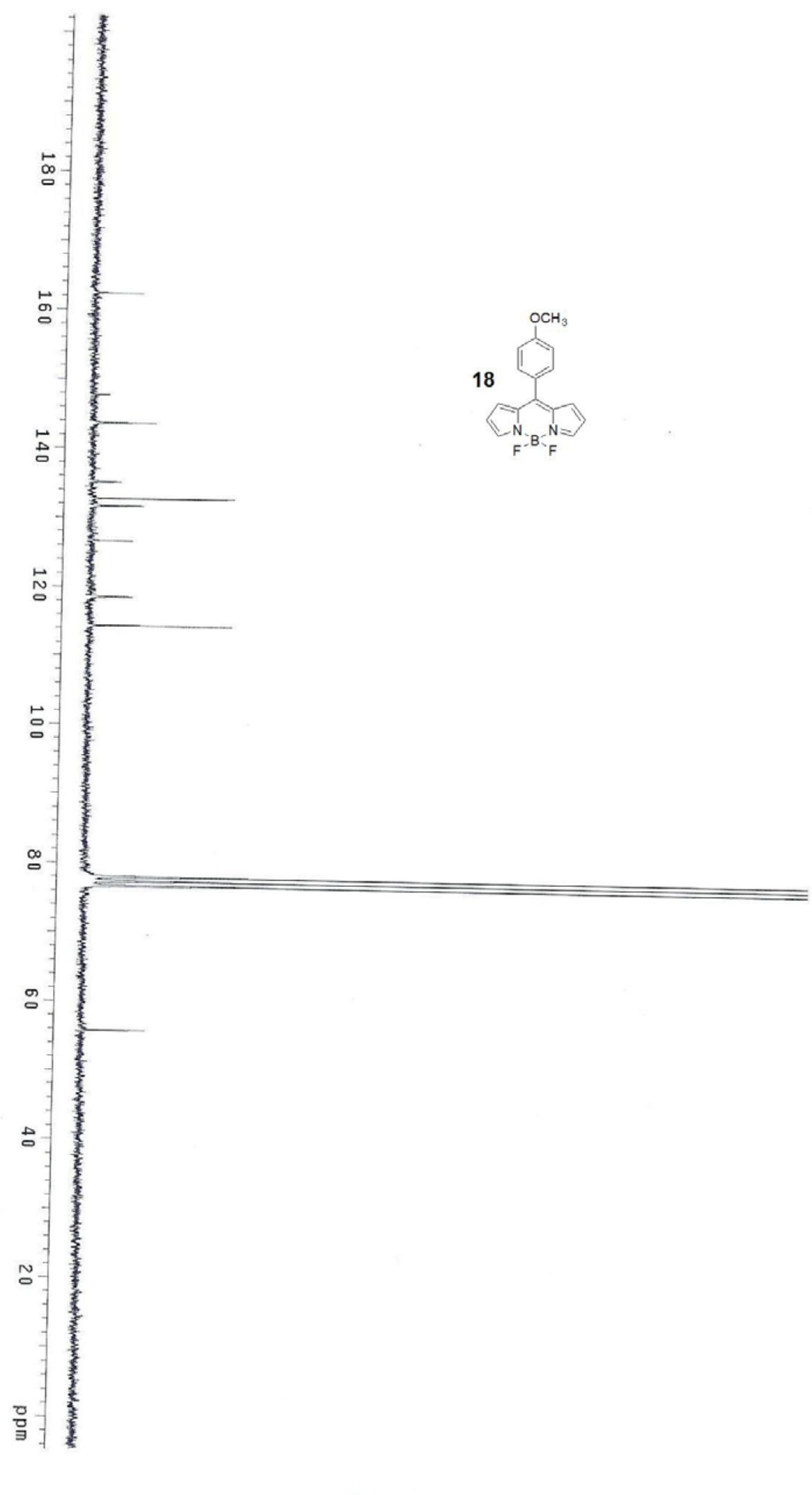




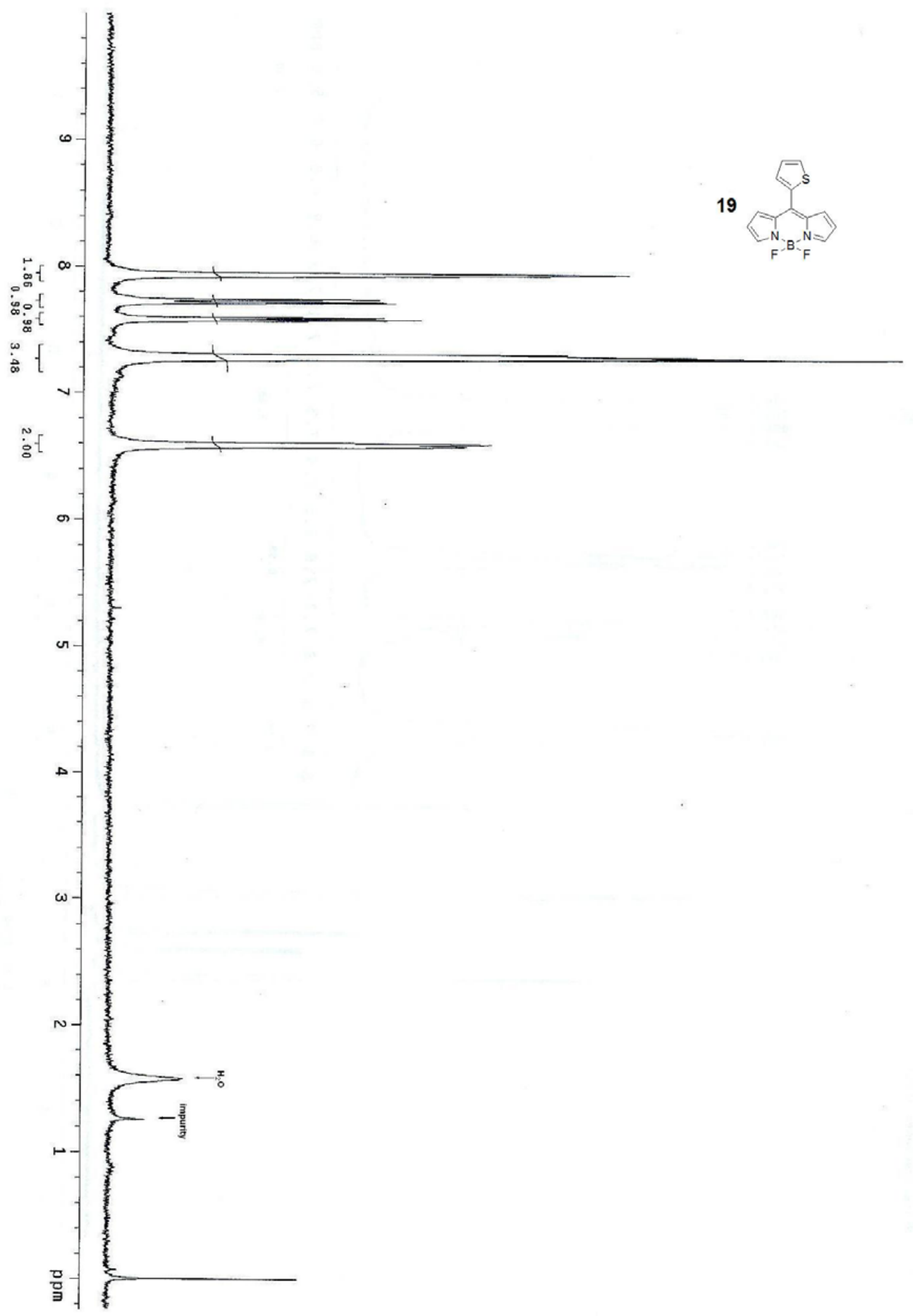




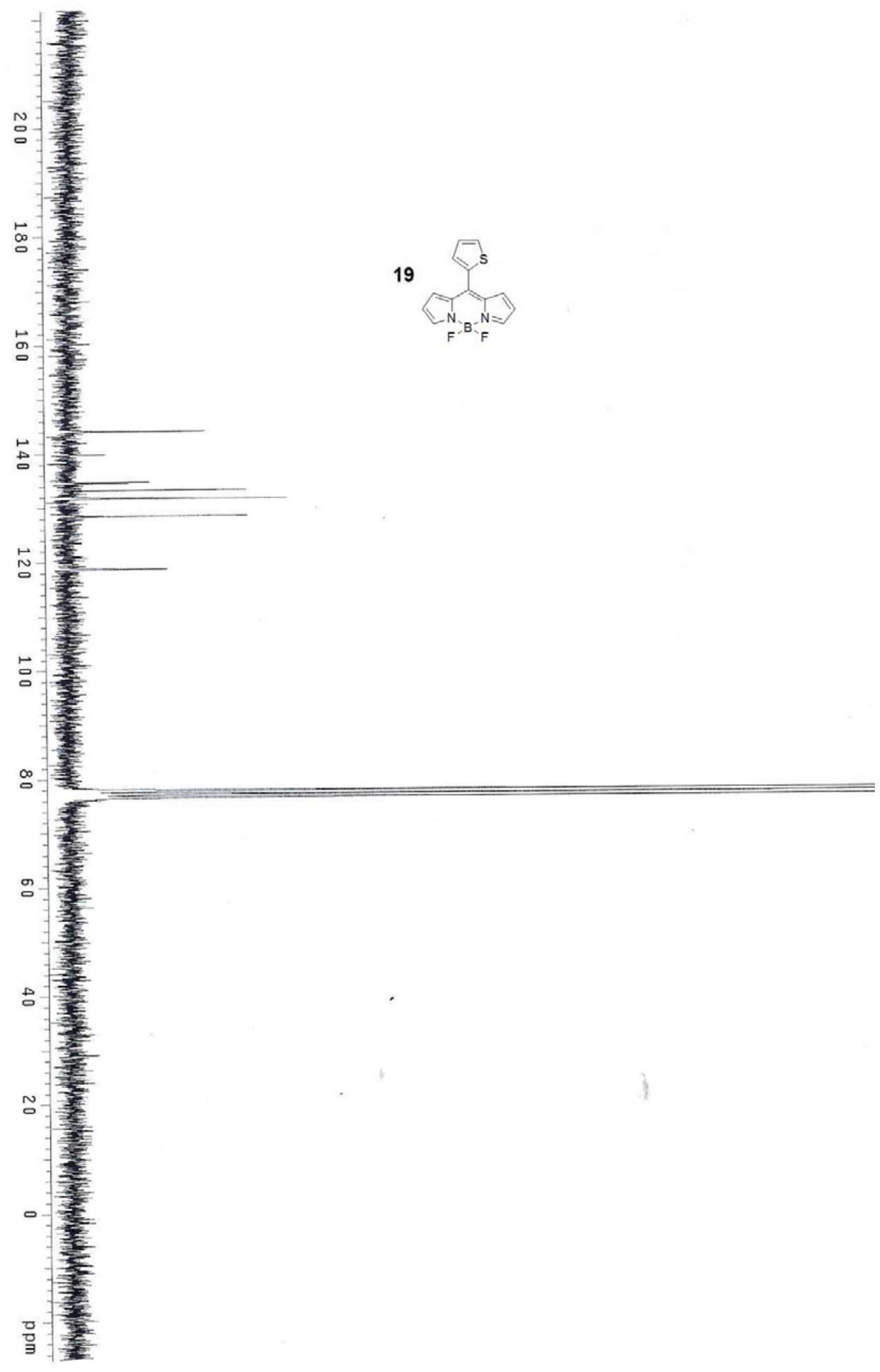




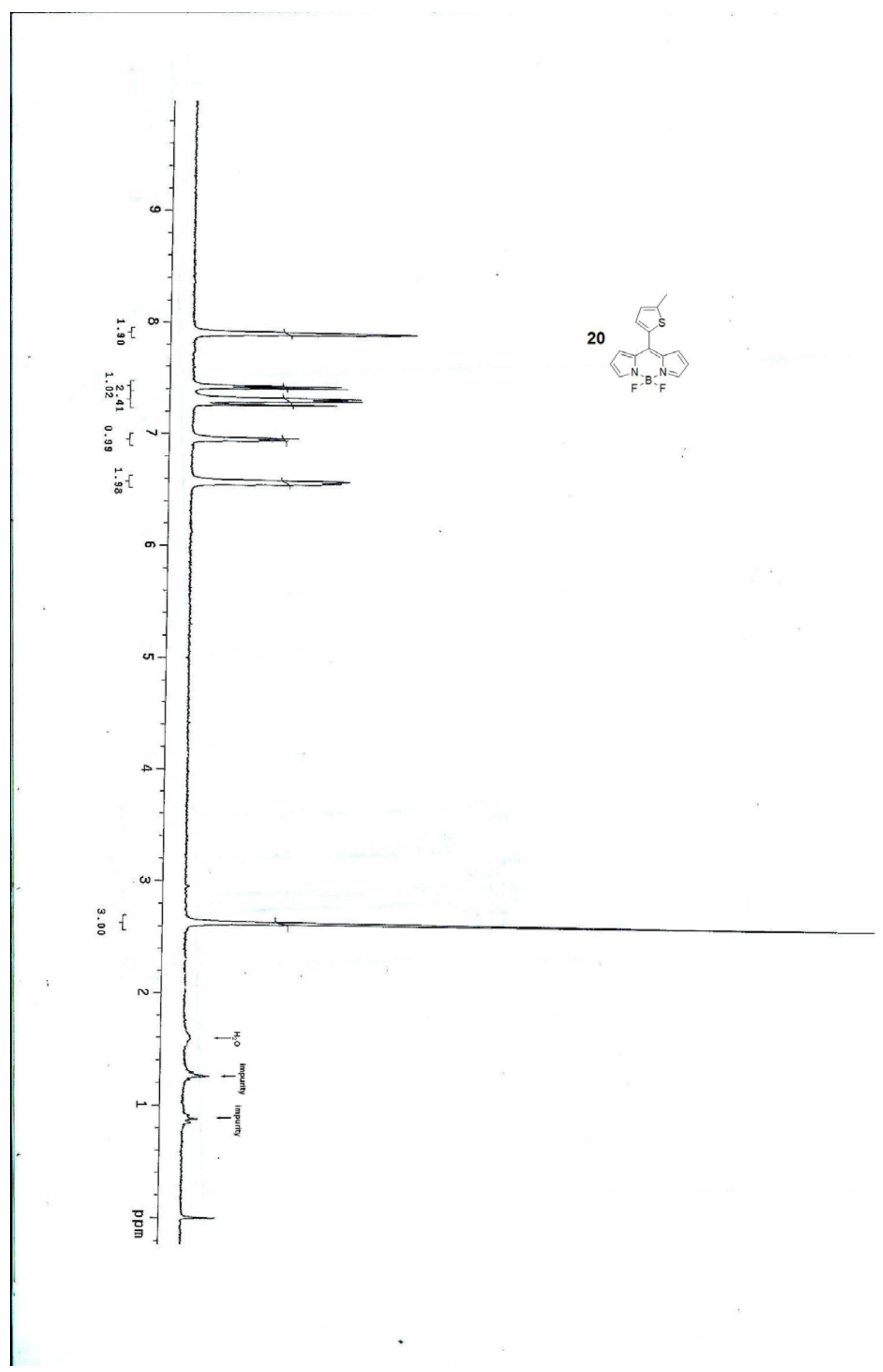




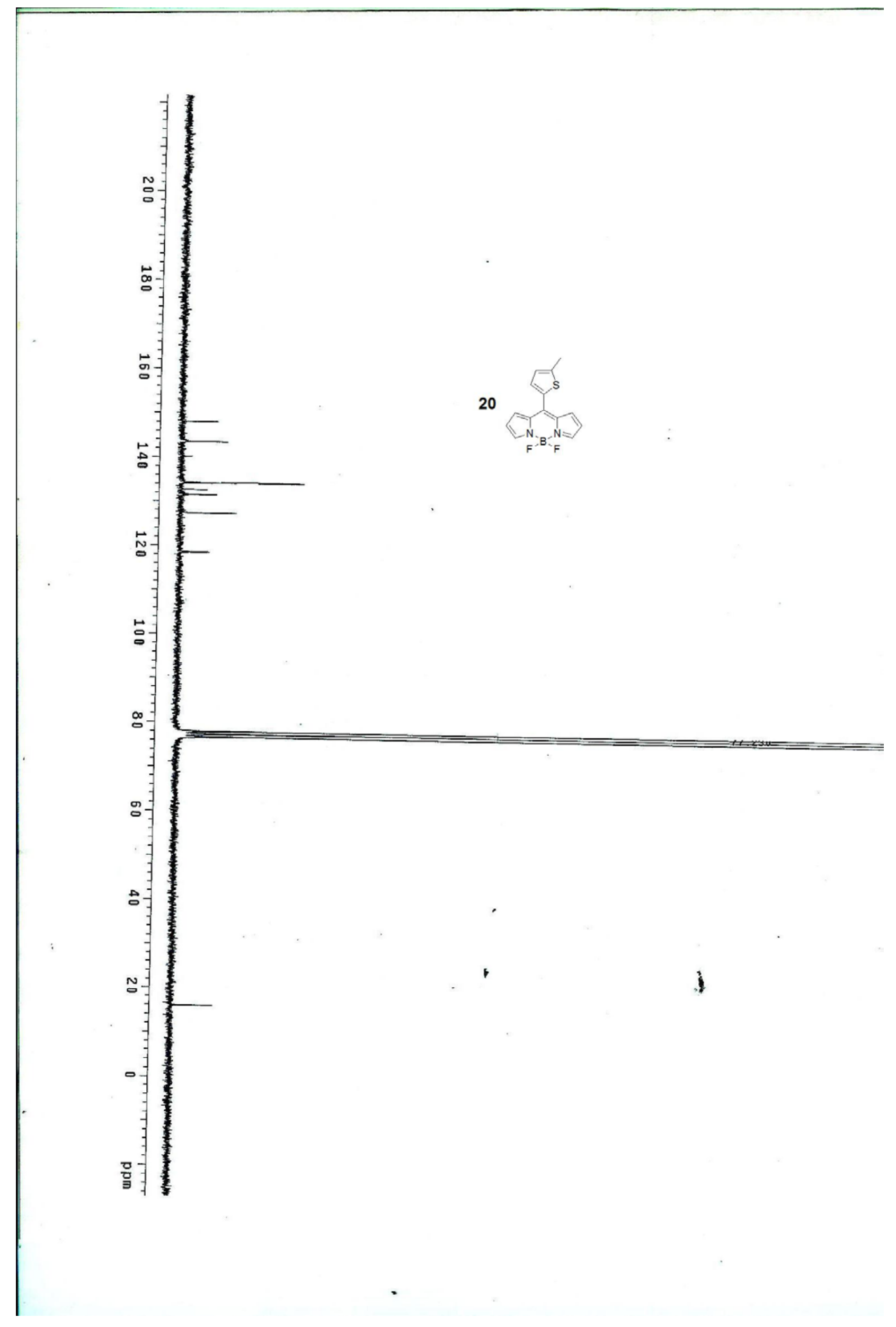




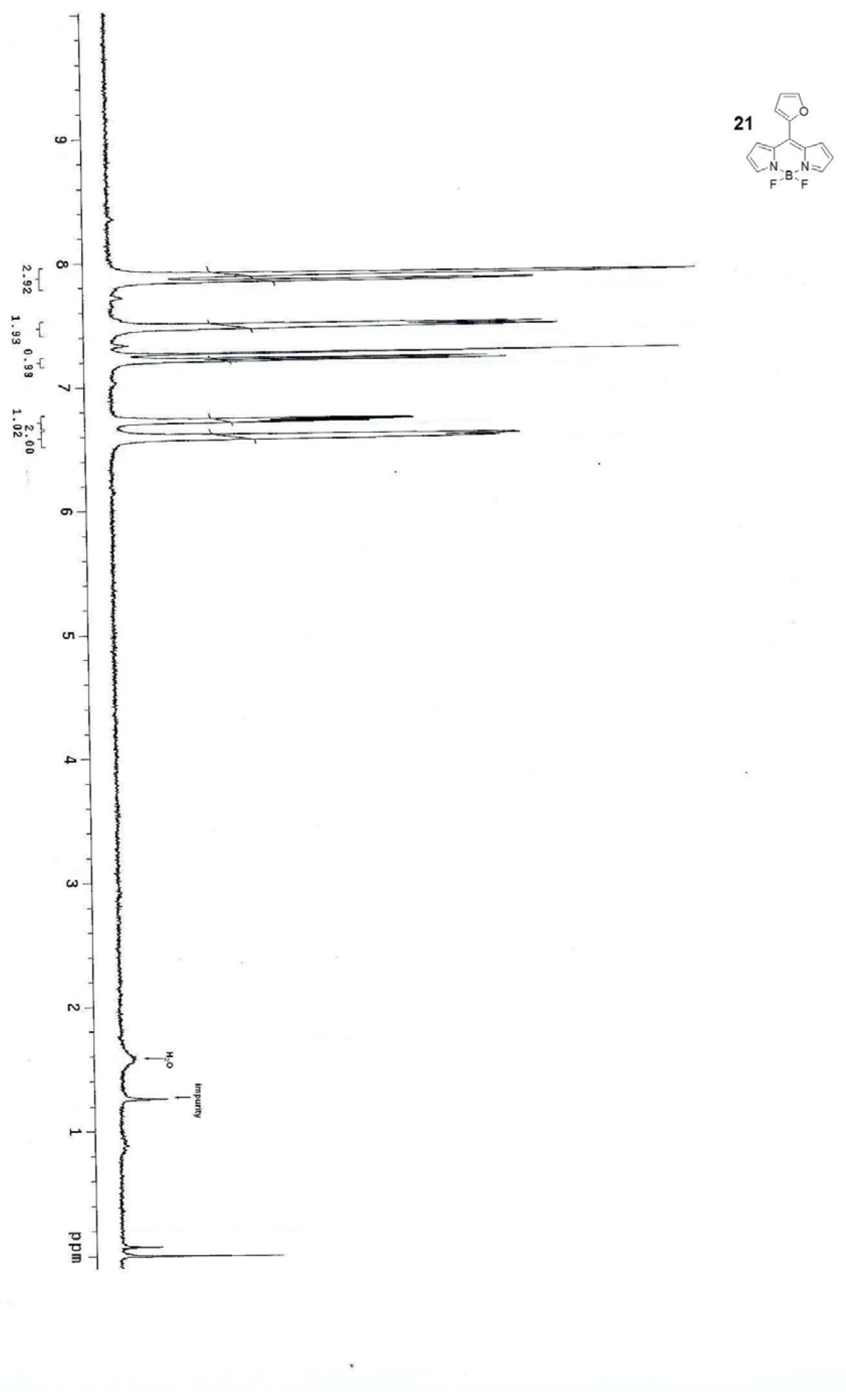




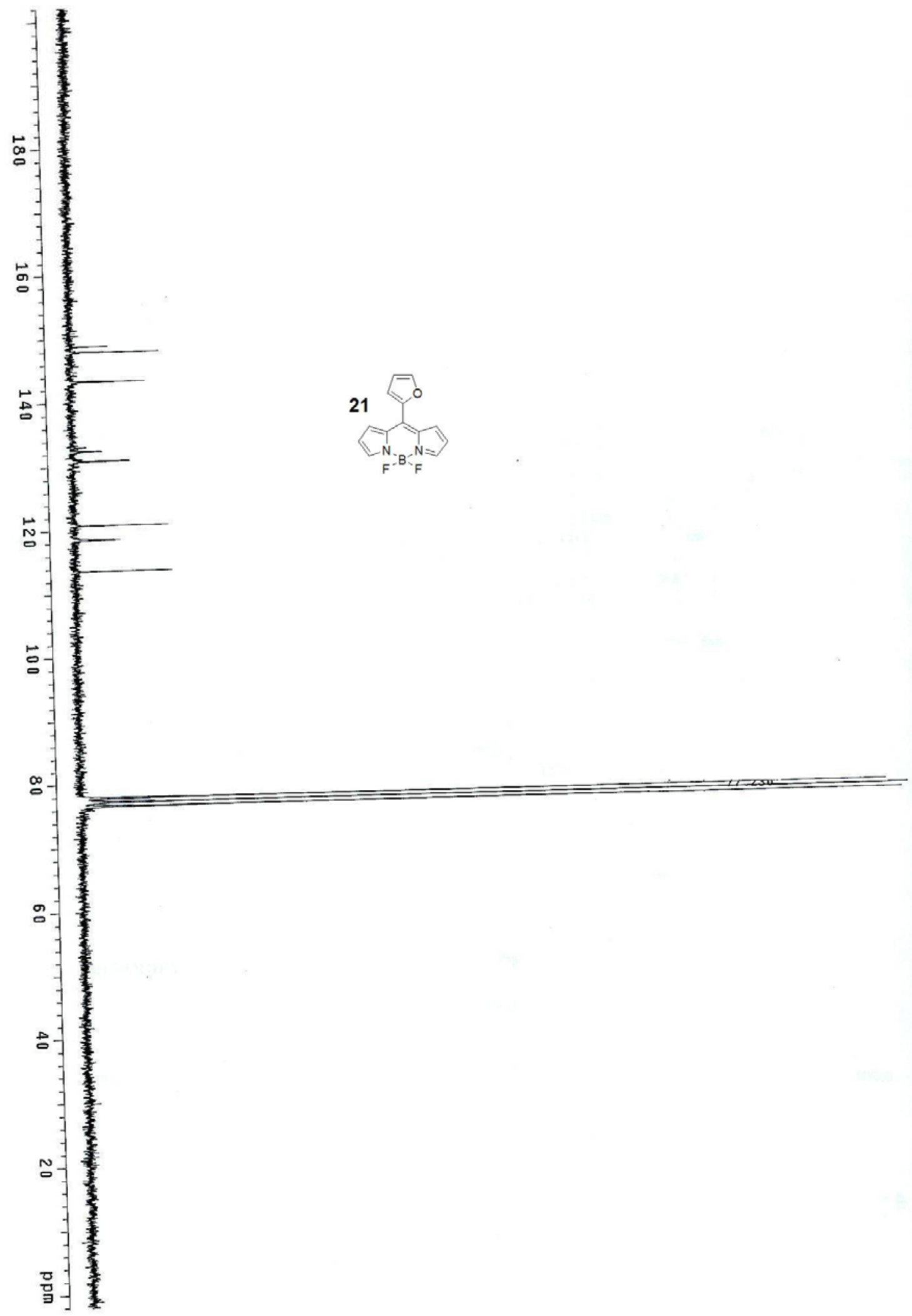


S51

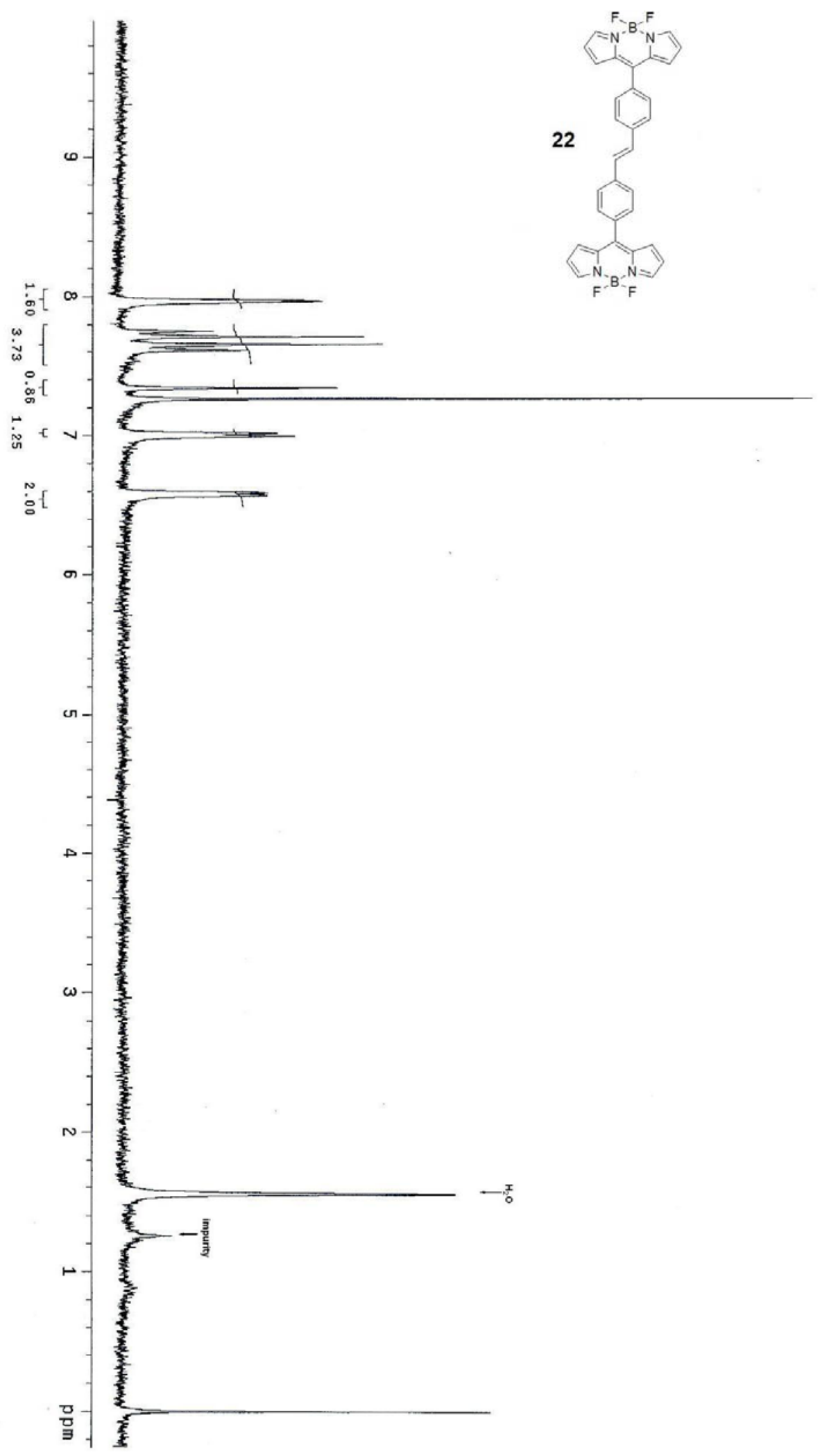




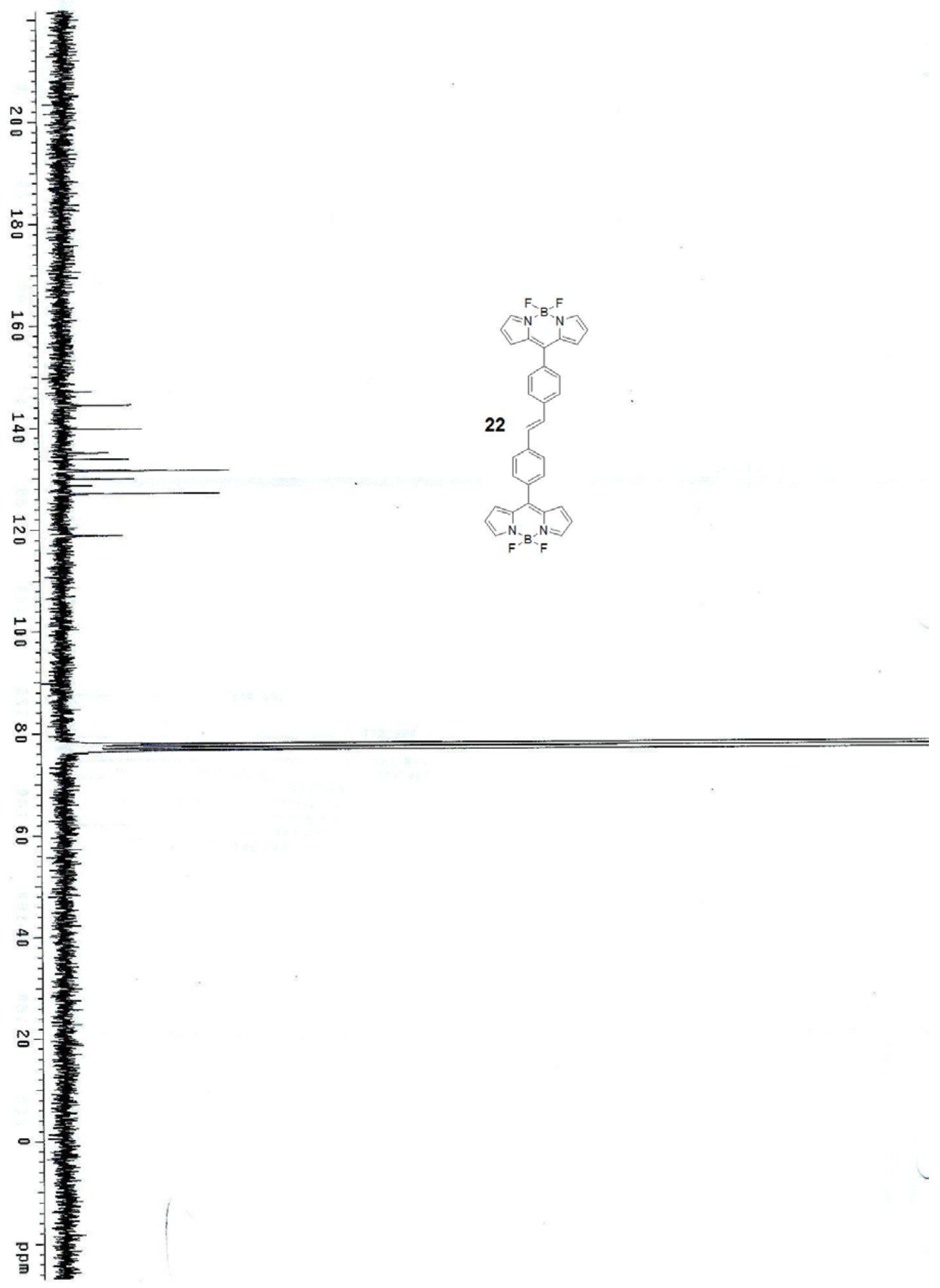




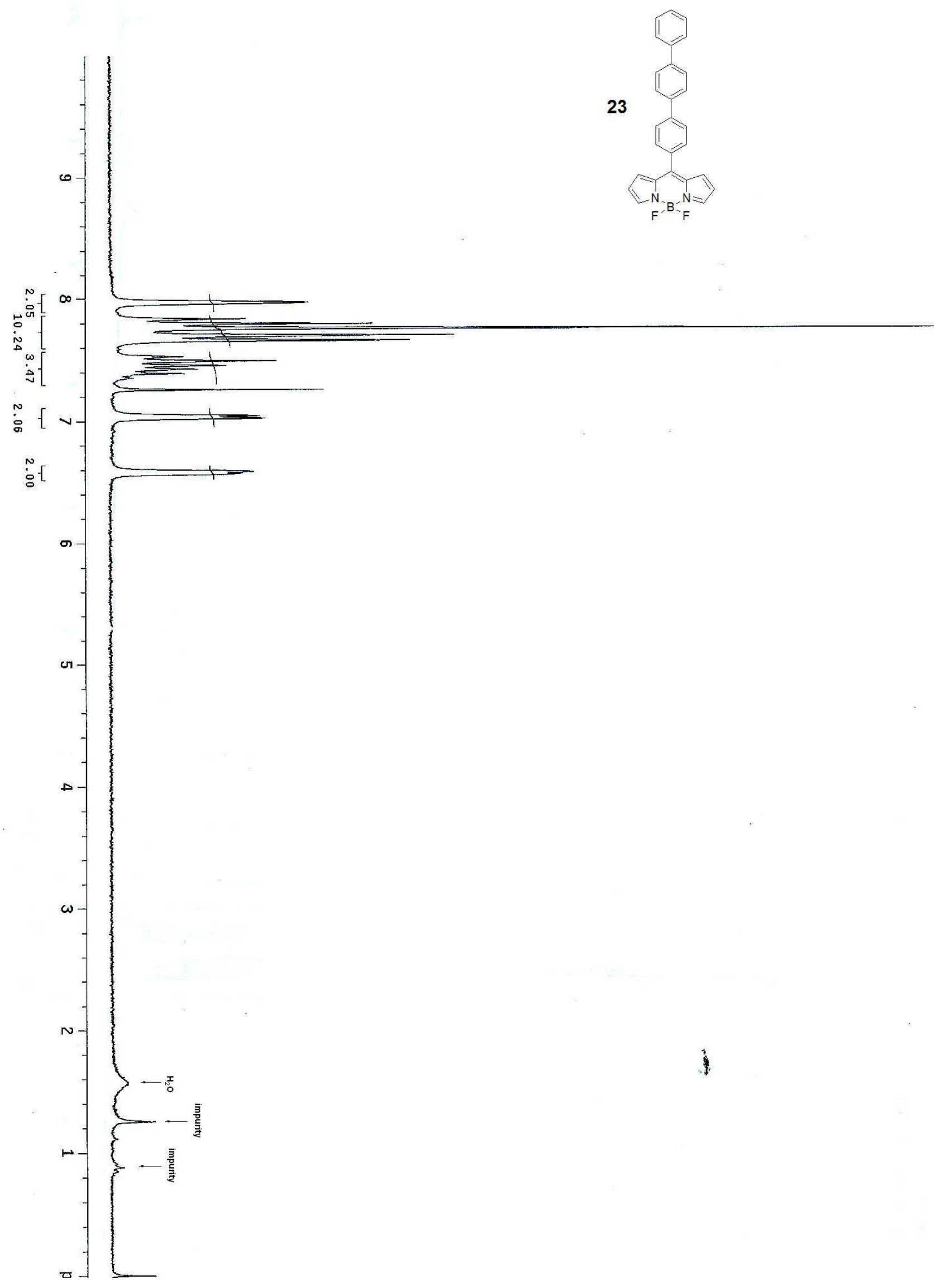




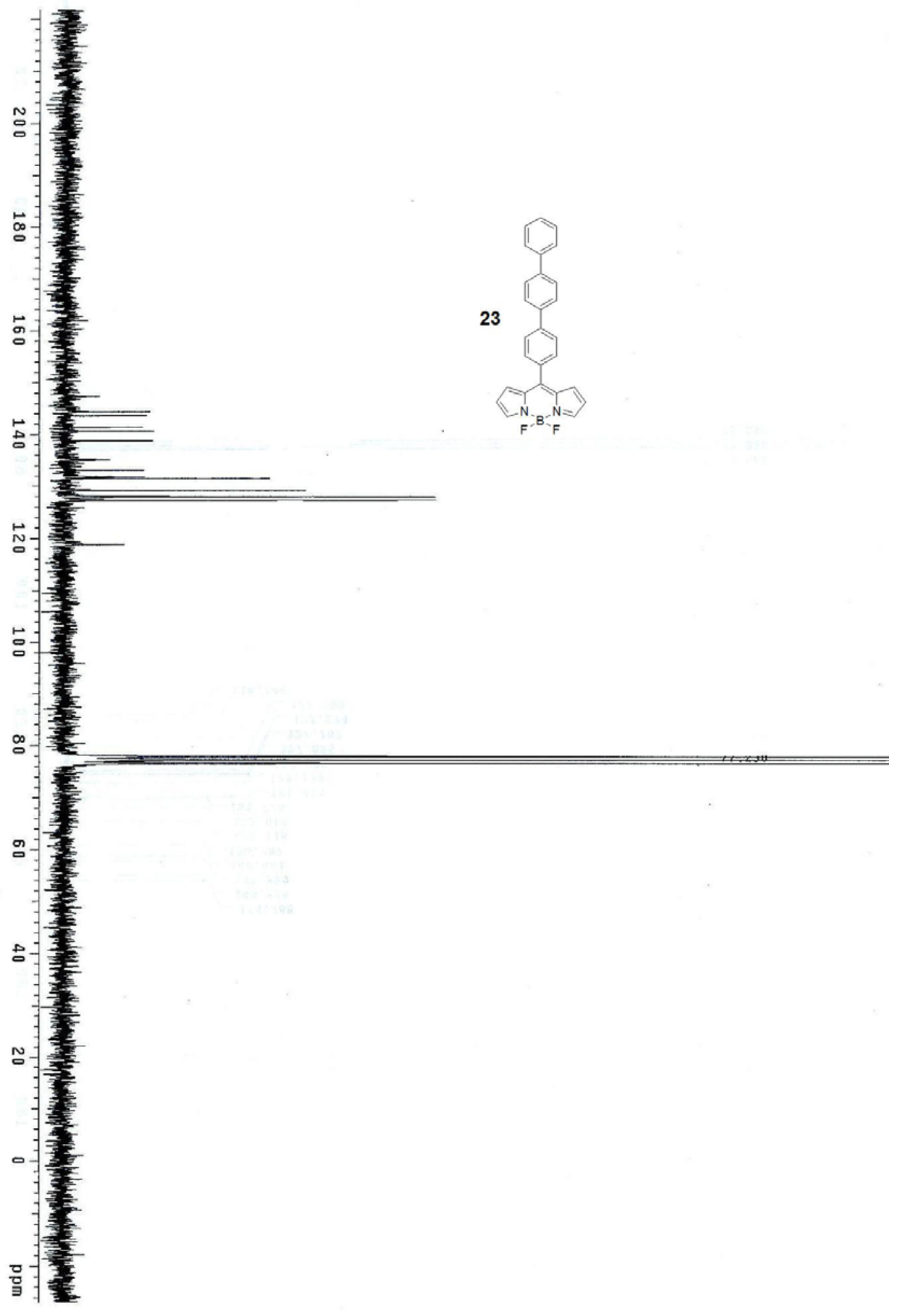




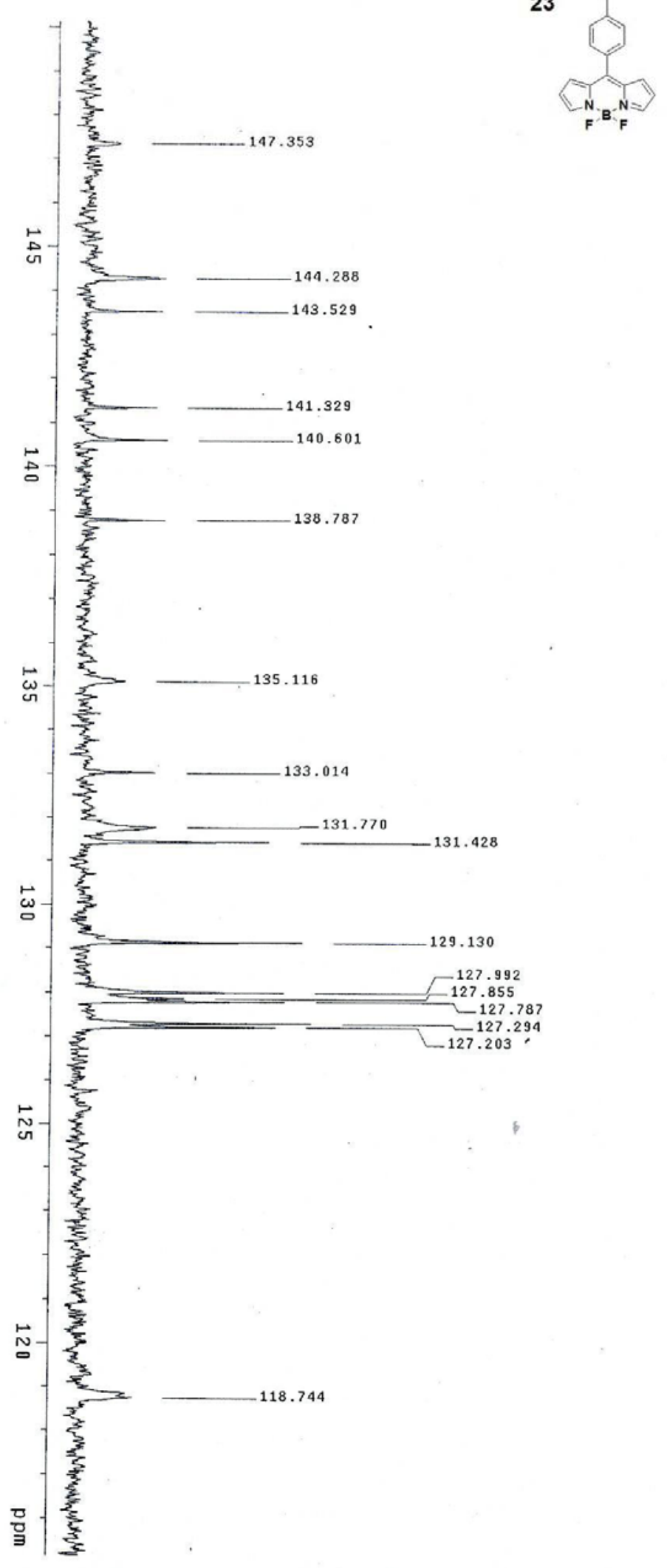

